\title{
Globale Zivilverfassungen: Alternativen zur staatszentrierten Verfassungstheorie
}

\author{
Gunther Teubner*
}

\section{Access to cyberspace?}

In einem spektakulären Prozeß versucht eine Gruppe von Globalisierungsgegnern, mit der Berufung auf die Meinungsfreiheit den Zugang zu einer Einrichtung des Internet gerichtlich zu erzwingen. Sie verklagt einen kommerziellen host provider, der auf seinen vernetzten Rechnern content providers die Möglichkeit anbietet, websites zu errichten. Seit längerem war der host provider schon in das Fadenkreuz von Staatsanwälten und privaten Sammelklägern geraten, da einige der websites Kinderpornographie und Nazipropaganda enthielten. Reagieren musste der provider zum einen auf die Entscheidung des Tribunal de Grande Instance de Paris, Beschluß vom 20. November 2000, wonach Yahoo Inc. den Zugang zu Versteigerungen von Naziobjekten für französische Nutzer sperren muß. ${ }^{1}$ Zum anderen reagierte der host provider auf neuere Trends zur öffentlich-privaten Co-regulierung des Internet in Europa und den Vereinigten Staaten, welche die Haftung des provider von seiner Kooperation mit staatlichen Instanzen abhängig machen. $^{2}$ Darauf sperrt der provider mit elektronischen Mitteln alle die websites, für die er das Prozessrisiko strafrechtlicher oder zivilrechtlicher Natur als zu hoch einschätzt. Von der Sperre sind auch als radikal eingestufte politische Gruppierungen betroffen. Mit einer Zivilklage versucht die Gruppe der Globalisierungsgegner nun, den Zugang zu den websites des host provider zu erzwingen.

Der Fall bündelt wie in einem Brennglas eine Fülle von fundamentalen Problemen, welche die Digitalisierung der Kommunikation aufwirft. Nicht nur technische juristische Fragen einer Prüfungspflicht für private provider, einer Zensur mißliebiger Inhalte durch Private, eines Aufnahmeanspruchs in verschieden Einrichtungen des Internet, der Geltung und Durchsetzung nationaler Normen im transnationalen Internet und der Drittwirkung von Grundrechten im cyberspace stehen zur Debatte. ${ }^{3}$ Vielmehr stellt sich die grundsätzlichere Frage eines universalen politischen Zugangsrechts zur digitalen Kommunikation, letztlich aber das Problem der Exklusion aus globalen Kommunikationsvorgängen. Im Hintergrund steht die Theoriefrage, ob es in der Entwicklungslogik funktionaler Differenzierung liegt, daß sich den binären Codes der globalen Funktionssysteme die Differenz von Inklusion/Exklusion überordnet. ${ }^{4}$ Wird Inklusion/Exklusion zum Metacode des 21. Jahrhunderts, der alle anderen Codes mediatisiert, zugleich aber die funktionale Differenzierung selbst untergräbt und mit der Brisanz des Ausschlusses ganzer Bevölkerungsgruppen andere gesellschaftspolitische Probleme dominiert?

* Prof. Dr. jur. M.A. (Berkeley) Professor für Privatrecht und Rechtssoziologie Universität Frankfurt und Centennial Visiting Professor London School of Economics.

Für kritisch-konstruktive Mitarbeit danke ich Gralf-Peter C a ll i e s s und Vaios K a r a v a s.

1 TGI Paris, Ordonnance de réferé du 20 nov. 2000, http://www.juricscom.net/txt/jurisfr/cti/tgiparis20001120.htm Dieses Urteil bestätigte das frühere Urteil vom 22.5.2000, mit dem Yahoo aufgefordert wurde, den Zugang zu Versteigerungsseiten von Nazimemorabilien zu unterbinden; TGI Paris, Ordonnance de réferé du 22 mai 2000 , http://222.juriscom.net/txt/jurisfr/cti/tgiparis20000522.htm

2 USA: 1990 Protection of Children from Sexual Predators Act, Section 42 U.S.C. $§ 13032 ; 1998$ Digital Millenium Copyright Act, 17 U.S.C. 512 (C). Europa: Richtlinie 2000/31.

Zu diesen Fragen, insbesondere zur Meinungsfreiheit im Internet Frydman, Benoit und Rorive, Isabelle (2002) "Regulating Internet Content through Intermediaries in Europe and the USA" Zeitschrift für Rechtssoziologie 23, 41-59; Holznagel, Bernd (2002) "Meinungsfreiheit oder Free Speech im Internet: Unterschiedliche Grenzen tolerierbarer Meinungsäußerungen in den USA und Deutschland" Archiv für Presserecht 9, 128-133; Hol z n a g el, Bernd (2000) "Responsibility for Harmful and Illegal Content as well as Free Speech on the Internet in the United States of America and Germany" In: Engel, Christoph und Keller, Kenneth H. (Hg.), Governance of Global Networks in the Light of Differing Local Values Baden-Baden: Nomos, 9-42; P i c hle r, Rufus (1999) "Meinungsfreiheit, Kunstfreiheit und neue Medien: Zwischen interessengerechter Auflösung von Rechtsgutkollisionen und 'Zensur'" Archiv für Presserecht 5/6, 429-439; Golds t o ne, David J. (1998) "A Funny Thing Happened on the Way to the Cyber Forum: Public vs. Private in Cyberspace Speech" Colorado Law Review 69, 1-70.

${ }^{4}$ Dazu im Rahmen einer Theorie der Weltgesellschaft Luhmann, Niklas (1993) Das Recht der Gesellschaft. Frankfurt: Suhrkamp, $582 \mathrm{ff}$. Zu Exklusion/Inklusion in systemtheoretischer und poststrukturalistischer Perspektive St äh e li, Urs und S t i c h w e h, Rudolf (Hg.) (2002) Exclusion and Socio-Cultural Identities: Systems Theoretical and Postructuralist Perspectives. Stuttgart: Lucius \& Lucius. 
Aus den vielen Problemen, die unser Rechtsfall aufwirft, möchte ich eine Frage herausgreifen: Wie reagiert die Verfassungstheorie auf die Herausforderungen, die sich aus den aktuellen drei großen Trends - Digitalisierung, Privatisierung und globaler Vernetzung - für das Problem der Inklusion/Exklusion ergeben? So sollte man im Unterschied zur Staatsverfassungsfrage des 18. und 19. Jahrhunderts die heutige "Verfassungsfrage" formulieren. Ging es damals in Deutschland um die Disziplinierung der absoluten politischen Gewalt durch ihre Rechtsbindung, so geht es heute um die Disziplinierung ganz anderer gesellschaftlicher Dynamiken. Dies ist in erster Linie eine Frage für die Theorie. Gelingt es ihr, die maßgeblichen Differenzen herauszuarbeiten, mit deren Hilfe sich der Verfassungsbegriff der nationalstaatlichen Tradition zeitgemäß generalisieren und respezifizieren läßt? Kann man also die Traditionen der Nationalstaatsverfassung fruchtbar machen und sie zugleich so verändern, daß sie den neuen Problemlagen der Digitalisierung, Privatisierung und globalen Vernetzung gerecht werden ${ }^{5}$

\section{Reaktionen der Verfassungstheorie}

Zeitgemäße Generalisierung und Respezifikation - an diesem Problem arbeiten sich vergeblich jene ehrgeizigen Versuche $a b$, die eine universale Weltverfassung jenseits des Nationalstaates postulieren. Dies betrifft juristische Bemühungen, die Charta der Vereinten Nationen zu einem Weltverfassungsrecht zu stilisieren, das von der international community in Geltung gesetzt sei und die Ausübung von weltpolitischer Gewalt legitimiere. ${ }^{6}$ Das gilt aber auch für eine Reihe von philosophischen Anstrengungen, die über Kant hinaus eine universalistische Weltverfassung denken, wonach die weltweite Einführung neuer Institutionen und Verfahren dazu dienen soll, ein föderatives Zentrum und Forum einer gemeinsamen Weltinnenpolitik einzurichten. ${ }^{7}$ All diesen Versuchen ist der Vorwurf zu machen, daß sie den traditionellen Verfassungsbegriff für heutige Verhältnisse nicht weit genug generalisieren und nicht sorgfältig genug respezifizieren, sondern statt dessen nationalstaatliche Verhältnisse unkritisch auf die Weltgesellschaft übertragen. Insbesondere bleibt unbedacht, welche Veränderungen ein Verfassungsbegriff in bezug auf Souveränität, organisiertes Kollektiv, Entscheidungshierarchie, organisierte Interessenaggregation und demokratische Willensbildung durchmachen müßte, wenn auf der Weltebene ein Äquivalent des Staates nicht aufzufinden ist. ${ }^{8}$

Realistischer schon setzen Versuche an, die eine deutliche Dissoziierung von Staat und Verfassung in Betracht ziehen und ausdrücklich eine globale Verfassung ohne Weltstaat denken. Von dieser innovativen Konstruktion ist jüngst ausgiebig in der Europaverfassungsdebatte Gebrauch gemacht worden, aber auch auf der Weltebene sucht man verfassungsrechtliche Elemente im laufenden Prozeß einer internationalen Politik, die über keine Zentralinstanz als Subjekt/Objekt einer Verfassung verfügt, aufzuspüren. ${ }^{9}$ Besonders der Versuch, in dem Nebeneinander von Nationalstaaten eine segmentäre Zweitdifferenzierung der Weltpolitik zu sehen und in deren Interaktion eine spontane Ordnung sekundärer Natur, eine "Weltverfassung der Freiheit", zu identifizieren, gibt einer so respezifizierten Weltverfassung als struktureller

5 Eindringlich zur Vermittlung von historischen Erfahrungen und Zukunftschancen im Grenzbereich Staat/Gesellschaft für globales Recht Zumbansen, Peer (2001) "Spiegelungen von 'Staat und Gesellschaft': Governance-Erfahrungen in der Globalisierungsdebatte" In: Anderheiden, Michael, Huster, Stefan und Kirste, Stephan (Hg.), Globalisierung als Problem von Gerechtigkeit und Steuerungsfähigkeit des Rechts Stuttgart: Steiner,13-40.

6 Ausführlich Fassbender, Bardo (1998) "The United Nations Charter as Constitution of the International Community" Columbia Journal of Transnational Law 37, 529- 619; D u p u y, Pierre-Marie (1997) "The Constitutional Dimension of the Charter of the United Nations Revisited" Max Planck Yearbook of United Nations Law 1, 1-33.

${ }^{7} \mathrm{H}$ öffe, Otfried (2001) "Königliche Völker": Zu Kants kosmopolitischer Rechts- und Friedenstheorie. Frankfurt: Suhrkamp; Haberma s, Jürgen (1998) Die postnationale Konstellation: Politische Essays. Frankfurt: Suhrkamp; R a w I s, John (1993) "The Law of Peoples" In: Shute, Stephen und Hurley, Susan (Hg.), On Human Rights: The Oxford Amnesty Lectures New York: Basic Books.

${ }^{8}$ Eine glänzende Kritik der "großen normativen Phantasmagorien" einer Politik der Weltgesellschaft findet sich bei S ch üt z, Anton (1997) "The Twilight of the Global Polis: On Losing Paradigms, Environing Systems, and Observing World Society" In: Teubner, Gunther (Hg.), Global Law Without A State Aldershot: Dartmouth Gower, 257-293.

${ }_{9}$ Zur europäischen Debatte J o erge s, Christian, M e n y, Yves und W e i l e r, Joseph H. H. (Hg.) (2000) What Kind of Constitution for What Kind of Polity? Responses to Joschka Fischer (Florence: Robert Schuman Centre, 2000). Firenze:Robert Schuman Centre; Di F a b i o, Udo (2000) "Eine europäische Charta" Juristenzeitung 55, 737-743; B o g d a n d y, Armin von (1999) Supranationaler Föderalismus als Wirklichkeit und Idee einer neuen Herrschaftsform: Zur Gestalt der Europäischen Union nach Amsterdam. Baden-Baden: Nomos; d e r s ., "Zweierlei Verfassungsrecht" Der Staat 39 (2000) 163 ff.; zur Verfassung der internationalen Gemeinschaft U e r p m a n n , Robert (2001) "Internationales Verfassungsrecht" Juristenzeitung 56, 565-573; T o m u s c h a t, Christian (1993) "Obligations Arising for States Without or Against Their Will" Recueil des Cours, 195-374. 
Kopplung zwischen dezentraler Weltpolitik und Recht ganz andere Konturen. ${ }^{10}$ Doch geht auch hier die Generalisierung nicht weit genug, als daß sie der Dezentrierung des Politischen in der Weltgesellschaft gerecht werden könnte. Insbesondere hat eine solche Spontanverfassung der zwischenstaatlichen Beziehungen mit dem Problem zu kämpfen, ob und wie sie nicht-staatliche Akteure im internationalen Prozeß als Verfassungssubjekte einbeziehen kann.

An diesem Defizit setzen wiederum Positionen an, die traditionell nicht als Völkerrechtssubjekte anerkannte Akteure ausdrücklich als Verfassungssubjekte konstitutieren. ${ }^{11}$ Die Akteure sind zum einen internationale Organisationen, multinationale Unternehmen, internationale Gewerkschaften, Interessenverbände und Nichtregierungsorganisationen als Beteiligte an weltpolitischen Entscheidungsprozessen, zum anderen die vom Völkerrecht nur zögernd und marginal als Rechtssubjekte anerkannten Individuen als Träger von Grund- und Menschenrechten. Implizit erkennen solche Konzeptionen an, daß die Prozesse der Digitalisierung und der globalen Vernetzung maßgeblich von nicht-staatlichen Akteuren getragen werden, von deren Existenz auch eine Weltverfassung Kenntnis nehmen müßte. Die Frage ist aber, ob eine bloß personelle Erweiterung eines Konstitutionalisierungsprozesses hinreicht oder ob nicht ganz andere Strukturen und Prozesse einbezogen werden müßten.

Noch einen Schritt weiter schließlich gehen Ideen der horizontalen Wirkung von Grundrechten, die materielle Grundrechtspositionen nicht mehr nur gegenüber politischen Instanzen behaupten, sondern auch gegen gesellschaftliche Institutionen, insbesondere gegenüber Zentren wirtschaftlicher Macht in Anspruch nehmen. Staatlichen Institutionen werden entsprechende Schutzpflichten auferlegt, um Grundrechtsgefährdungen in staatsfernen Bereichen zu begegnen. ${ }^{12}$ Auch wenn diese Debatte im internationalen Bereich nur in den ersten Anfängen steht, indiziert sie doch angesichts der massiven Menschenrechtsverletzungen durch nicht-staatliche Akteure die Notwendigkeit einer Ausweitung der weltverfassungsrechtlichen Auseinandersetzungen über die bloßen zwischenstaatlichen Beziehungen hinaus. ${ }^{13}$

\section{These: Konstitutionalisierung ohne Staat}

Diese vier Entwürfe einer Globalverfassung stellen gegenüber den Konstruktionen der Verfassungstradition durchaus dramatische Erweiterungen dar, doch verdichtet sich der Eindruck, daß sie sich letztlich nicht von der Faszination von der Architektur des Nationalstaats lösen können, sondern deren offenkundige Unzulänglichkeiten nur mit allerlei Reparaturen, Anbauten, Umbauten, Unterkellerungen und Fassadenverzierungen - insgesamt mit bloßen Komplexifizierungen der Konstruktion statt eines Neubaus - auszugleichen suchen. Der Konstruktionsfehler aber liegt schon in der Sta atszentrierung der Verfassung. ${ }^{14}$ Bei allem Mut, die Verfassung in Richtung auf politische Globalität, in Hinblick auf einen zwischenstaatlichen Prozeß, über Einbeziehung gesellschaftlicher Akteure bis hin zur strukturellen Wirkung der Grundrechte in die Gesellschaft hinein umzudenken, bleiben sie doch fixiert auf ein Verständnis von Verfassung als Freisetzung und Beschränkung staatlich-politischen Handelns.

\footnotetext{
${ }^{10}$ In Anschluß an Hayek O e te r, Stefan (2000) "Internationale Organisation oder Weltföderation? Die organisierte Staatengemeinschaft und das Verlangen nach einer 'Verfassung der Freiheit" In: Brunkhorst, Hauke und Kettner, Matthias (Hg.), Globalisierung und Demokratie: Wirtschaft, Recht, Medien Frankfurt: Suhrkamp, 208-224.

${ }^{11}$ Zu dieser Option F i s c h e r - L e s c a n o, Andreas (2002b) "Globalverfassung: Verfassung der Weltgesellschaft" Archiv für Rechts- und Sozialphilosophie 88, 349-378, $371 \mathrm{ff} . ;$ W alk er, Neil (2002) "The Idea of Constitutional Pluralism" Modern Law Review 65, 317-359; W a I t e r, Christian (2001) "Constitutionalizing (Inter)national Governance: Possibilities for and Limits to the Development of an International Constitutional Law" German Yearbook of International Law 44, 170-201.

12 Ruffert, Matthias (2001) Vorrang der Verfassung und Eigenständigkeit des Privatrechts: eine verfassungsrechtliche Untersuchung zur Privatrechtswirkung des Grundgesetzes. Tübingen: Mohr Siebeck; J a r a s s , Hans D. (2001) "Die Grundrechte: Abwehrrechte und objektive Grundsatznormen. Objektive Grundrechtsgehalte, insbes. Schutzpflichten und privatrechtsgestaltende Wirkung" In: Badura, Peter und Dreier, Horst (Hg.), Festschrift 50 Jahre Bundesverfassungsgericht Tübingen: Mohr Siebeck, 35-53; P re e dy, Kara (2000) "Fundamental Rights and Private Acts: Horizontal Direct or Indirect Effect? - A Comment" European Review of Private Law, 125-133.

${ }_{13}$ Zur Grundrechtsdrittwirkung im europäischen Kontext Schindler, Dierk (2001) Die Kollision von Grundfreiheiten und Gemeinschaftsgrundrechten: Entwurf eines Kollisionsmodells unter Zusammenführung der Schutzpflichten- und Drittwirkungslehre. Berlin: Duncker-Humblot; C I a p h a m, Andrew (1996) Human Rights in the Private Sphere. Oxford: Oxford University Press; P a u s t, Jordan J. (2002) "Human Rights Responsibilities of Private Corporations" Vanderbilt Journal of Transnational Law 35, 801-825; M u chlinski, Peter T. (2001) "Human Rights and Multinationals: Is There a Problem?" International Affairs 77, 31-48.

${ }^{14} \mathrm{So}$ in wünschenswerter Deutlichkeit der Vertreter eines konstitutionellen Pluralismus W al k e r, Anm. 11.
} 
Sie sind dabei zugleich einer merkwürdigen Unterscheidung verhaftet, zwischen deren Polen sie unentwegt oszillieren. ${ }^{15}$ Zwar soll sich die Verfassung institutionell auf die politischen Prozesse beschränken, zugleich aber erhebt sie den Anspruch, auf diese Weise die Gesamtgesellschaft zu verfassen. In der politischen Organisation des Staates verfaßt sich die Nation. Dieses Oszillieren zwischen Politikverfassung und Gesellschaftsverfassung wird unbesehen auf die Weltgesellschaft übertragen. Wenn es nur gelingt, die Interaktion staatlichpolitischer Institutionen in den internationalen Beziehungen zu konstitutionalisieren, dann soll allein daraus eine der Weltgesellschaft angemessene Verfassung resultieren. War diese merkwürdige Unterscheidung schon im Nationalstaat problematisch, so ist sie in der Weltgesellschaft endgültig überholt. Was aber liegt im blinden Fleck der Unterscheidung? Eine umfassende Weltverfassung? Ein Netzwerk der Nationalstaatsverfassungen? Eine autonome Rechtsverfassung? Oder was sonst?

Gibt man, um den blinden Fleck auszuleuchten, die Staatszentrierung des Verfassungsbegriffs auf, so wird die Sicht auf die realen Möglichkeiten einer Konstitutionalisierung ohne Staat frei. Für Verfassungstheoretiker ist dies ein Tabubruch. Verfassung ohne Staat ist für sie allenfalls eine Utopie und eine schlechte dazu. ${ }^{16}$ Mit dieser Formel ist aber damit gerade nicht eine abstrakte normative Forderung für ferne, ungewisse Zukunften erhoben, sondern es wird ein Realtrend behauptet, der sich heute im globalen Maßstab beobachten läßt. Die These heißt: Emergenz einer Vielzahl von Zivilverfassungen. Die Verfassung der Weltgesellschaft verwirklicht sich nicht exklusiv in den Stellvertreter-Institutionen der internationalen Politik, sie kann aber auch nicht in einer alle gesellschaftlichen Bereiche übergreifenden Globalverfassung stattfinden, sondern sie entsteht inkrementell in der Konstitutionalisierung einer Vielheit von autonomen weltgesellschaftlichen Teilsystemen. ${ }^{17}$

Die im Internet tobenden Kämpfe um cyberanarchy, staatliche Regulierung und Kommerzialisierung sind verfassungspolitische Konflikte ersten Ranges, in deren chaotischem Verlauf nichts anderes als das Organisationsrecht einer Digitalverfassung allmählich Konturen gewinnt. ${ }^{18}$ Nicht von ungefähr benutzt die berühmt-berüchtigte Declaration of the Independence of Cyberspace das verfassungspolitische Pathos der Gründerväter und erklärt gegenüber den

"Governments of the Industrial World, you weary giants of flesh and steel, I come from Cyberspace,

the new home of Mind. ..., the global social space we are building to be naturally independent of the

tyrannies you seek to impose on us. You have no moral right to rule us nor do you possess any methods of enforcement we have true reason to fear". ${ }^{19}$

Zunehmend werden vor nationalen Gerichten und internationalen Schiedsgerichten Streitfälle getragen, in denen über die Geltung von Grundrechten im cyberspace entschieden wird. ${ }^{20}$ Eines der vielen Grundrechtsprobleme der Internetverfassung stellt sich in unserem Rechtsfall. Ob ein Zugangsanspruch gegenüber einem host provider im Internet besteht oder nicht, ist aufgrund einer Diskussion der Inklusionsprinzipien digitaler Kommunikation zu entscheiden. ${ }^{21}$ Nicht die auf

\footnotetext{
${ }^{15}$ Zu diesem Argument L u h m a n n, Niklas (2000) Die Politik der Gesellschaft. Frankfurt: Suhrkamp, 201, 207 f., 217.

${ }^{i 6}$ Besonders ausgeprägt bei G r i m m, Dieter (1995) "Braucht Europa eine Verfassung?" Juristenzeitung 50, 581591.

${ }^{17}$ Eine ähnliche These vertreten W alker , Anm. 11, und W alt e r, Anm. 11, 188 ff.; es bleibt abzuwarten, ob sie auch eine radikalere Version des Rechtspluralismus, die eine Konstitutionalisierung ohne Staat voraussetzt, anerkennen würden, wenn es um private governance regimes geht.

${ }^{18}$ Vgl. die explizit verfassungsrechtlich geführte Debatte zwischen Lessig, Lawrence (1999) Code and Other Laws of Cyberspace. New York: Basic Books und J o h n s o n, David und P o s t, David (1998) "The New 'Civic Virtue' of the Internet: A Complex System Model for the Governance of Cyberspace" http://www.temple.edu/lawschool/dpost/Newcivicvirtue.html

${ }_{19}$ B a rlow, John P. (2002) Cyberspace Declaration of Independence.
} http://www.eff.org.//Publications/John Perry Barlow

${ }^{20}$ Zur Problematik der Drittwirkung der Grundrechte in den Domainstreitigkeiten des Internet siehe Go Id s t e i n , Adam (2002) "ICANNSucks.biz (And Why You Can't Say That): How Fair Use of Trademarks in Domain Names is Being Restrained, Fordham Intellectual Property", Media \& Entertainment Law Journal 12, 1151-1186; M u e II e r, Milton (2002) Ruling the Root - Internet Governance and the Taming of Cyberspace, $245 \mathrm{ff} . ; \mathrm{B} \mathrm{I} \mathrm{a} \mathrm{c} \mathrm{k} \mathrm{m} \mathrm{a} \mathrm{n,} \mathrm{Keith} \mathrm{(2001)} \mathrm{"The}$ Uniform Domain Name Dispute Resolution Policy: A Cheaper Way to Hijack Names and Supress Critics", Harvard Journal of Law \& Technology 15, 211-256; N a ja ri a n, David C. (2001) "Internet Domains and Trademarks Claims: First Amendment Considerations", Journal of Law \& Technology 41, $127 \mathrm{ff}$., wie auch unter http://www.idea.piercelaw.edu/articles/41/41_1/5.Najarian.pdf; Sorge n, Rebecca S. (2001) "Trademark Confronts Free Speech on the Information Superhighway: 'Cybergripers' Face a Constitutional Collision", Loyola of Los Angeles Entertainment Law Review 22, 115-152.

21 Vgl. die Entscheidungen des LG Bonn MMR 2000, 109 und des OLG Köln MMR 2001, 52, die der Parallelproblematik des Zugangs zu einem chat room eines providers mit einer merkwürdigen Mischung aus eigentumsrechtlichen und vertragsrechtlichen Denkfiguren gerecht zu werden suchen. Dazu La de u r, Karl-Heinz 
Machtakkumulation und policy-Formulierung zielenden Prinzipien einer politischen Verfassung, sondern die auf Kommunikationsfreiheit und ihre elektronischen Gefährdungen zielenden Prinzipien einer Internetverfassung, die ihrerseits im Laufe der Konstitutionalisierung erst noch zu erarbeiten sind, stellen die sedes materiae digitalverfassungsrechtlicher Normen dar. ${ }^{22}$ Die offene Frage in unserem Ausgangsfall ist, ob die neue privat-öffentliche Co-Regulierung es über ökonomische Anreize Privatunternehmen überlassen darf, die Grenzen der Meinungsfreiheit im Internet zu definieren. ${ }^{23}$

Um die Kampfzone auszuweiten: Von Seattle bis Genua finden in den Konferenzsälen und auf der Straße Kämpfe um eine Weltwirtschaftsverfassung statt, deren Ergebnisse den internationalen Institutionen Weltbank, IWF und WTO einen konstitutionellen Schub geben werden. Eine globale Wissenschaftsverfassung und eine Verfassung des globalen Gesundheitssystems formieren sich in den erregten wissenschaftsinternen und -externen Debatten über Embryonenforschung und reproduktive Medizin und sind auf der Suche nach wissenschafts- und medizinadäquaten Äquivalenten für traditionelle staatsbezogene Grundrechte. Und seit dem 11. September 2001 mehren sich die Versuche, die Auseinandersetzungen zwischen den Weltreligionen in rechtlich verfaßten Einrichtungen des interreligiösen Dialogs stärker zu institutionalisieren.

\section{Drei Entwicklungstrends}

Eine solche Forderung, von der einen Staatsverfassung der Nation auf die vielen Zivilverfassungen der Weltgesellschaft umzustellen, wirft natürlich sofort die Frage auf, welche Umstände es rechtfertigen, das nationalstaatliche Modell einer exklusiv politischen Verfassung, das sich anscheinend über Jahrhunderte bewährt hat, für die Weltgesellschaft zu verwerfen. Sehr schematisch und reichlich verkürzt will ich drei Entwicklungstrends skizzieren, die dem staatszentrierten Verfassungsdenken den Boden $\mathrm{zu}$ entziehen und einen "societal constitutionalism" empirisch und normativ plausibel zu machen geeignet sind.

\section{Diagnose I: Dilemma der Rationalisierung}

Hier liefert die von dem amerikanischen Soziologen David Sciulli entwickelte "theory of societal constitutionalism" erste Anhaltspunkte. ${ }^{24}$ Ausgehend von dem von Max Weber eindringlich analysierten Dilemma der Rationalisierungsprozesse der Moderne stellt S ciulli die Frage nach existierenden Gegenkräften zu einem massiven evolutionären Drift, der sich in vier Schüben manifestiert:

(1) Fragmentierung der Handlungslogiken mit den Folgen hochgetriebener Differenzierung, Pluralisierung und wechselseitige Abschottung separierter Sinnsphären: Jeder gesellschaftliche Handlungsbereich entwickelt seine eigenwillige formale Rationalität, die sich in einem unauflöslichen Konflikt mit den Rationalitäten anderer Bereiche befindet.

(2) Dominanz instrumenteller Kalkulation als der einzigen Rationalität, die bereichsübergreifend Anerkennung findet: Angesichts der Rationalitätenkollision der Moderne setzt sich hauptsächlich in Wirtschaft und Politik, aber zunehmend auch in den anderen Handlungssektoren allein die Logik des instrumentellen Kalkulierens durch.

(3) Umfassende Ablösung informeller Koordination durch bürokratische Organisation: In allen Lebensbereichen verbreiten sich zunehmend formale hierarchisch strukturierte und mit Expertenwissen ausgestattete Organisationen als Träger der formalen Rationalität aus.

(2002) "Rechtsfragen des Ausschlusses von Teilnehmern an Diskussionsforen im Internet: Zur Absicherung von Kommunikationsfreiheit durch netzwerkgerechtes Privatrecht" Multimedia und Recht 5, 787-792, der explizit ein netzwerkgerechtes Privatrecht fordert.

${ }^{22}$ Für eine internetadäquate Umformulierung des Grundrechts der Meinungsfreiheit im Zusammenhang der Konflikte um Internet-Domains, Karavas, Vaios und Teubner, Gunther (2003 im Erscheinen) "http://www.CompanyNameSucks: Grundrechte gegenüber 'Privaten' im autonomen Recht des Internet?" In: HoffmannRiem, Wolfgang und Ladeur, Karl-Heinz (Hg.), Innovationsoffene Regulierung des Internet Baden-Baden: Nomos,.

${ }^{23} \mathrm{Frydm}$ an und Rorive, Anm. 3.

${ }^{24}$ S c i u ll i , David (1992) Theory of Societal Constitutionalism. Cambridge: Cambridge University Press, 42 ff.; vgl. auch de r s . (2001) Corporate Power in Civil Society: An Application of Societal Constitutionalism. New York: New York University Press; d e r s . (1994) "The Critical Potential of the Common Law Tradition" Columbia Law Review 94, 10761124; d e r s . (1988) "Foundations of Societal Constitutionalism: Principles from the Concepts of Communicative Action and Procedural Legality" British Journal of Sociology 39, 377-407. 
(4) Zunehmende Einschließung in die "Gehäuse der Hörigkeit der Zukunft": Besonders außerhalb der Politik nimmt in den gesellschaftlichen Bereichen die formale Organisierung des Handelns zu, dessen Auswirkungen zu einer umfassenden Regelorientierung des Individuums führen.

Dieser Drift endet gesellschaftsweit unausweichlich in einer Situation intensiver Konkurrenz um Machtpositionen und sozialen Einfluß, hochformalisierter sozialer Kontrolle und politischem und sozialem Autoritarismus. Sie hat darüber hinaus dilemmatischen Charakter, weil jeder bewußte Versuch, kollektive Kontrolle über den Drift zu gewinnen, sich selbst in dieser Logik verfängt und den Drift nur verstärkt. ${ }^{25}$

Die einzige gesellschaftliche Dynamik, die diesem evolutionärem Drift in der Vergangenheit effektiv entgegengearbeitet hat und in Zukunft Widerstand leisten kann, ist nach Sciulli in den Institutionen eines societal constitutionalism zu finden:

"Only the presence of institutions of external procedural restraint (on inadvertent or systemic exercises of collective power) within a civil society can account for the possibility of a nonauthoritarian social order under modern conditions." ${ }^{16}$

Entscheidend sei die soziale Institutionalisierung der Prozeduren von (im Sinne der rational choice) nicht-rationalen Normierungen, die sich empirisch in collegial formations, d.h. in den spezifischen Organisationsformen der Professionen und anderer normproduzierender und deliberativer Institutionen identifizieren lassen,

"it is typically found not only within public and private research institutes, artistic and intellectual networks, and universities, but also within legislatures, courts and commissions, professional associations, and for that matter, the research divisions of private and public corporations, the rulemaking bodies of nonprofit organizations, and even the directorates of public and private corporations." ${ }^{27}$

Normative Konsequenz ist, die Autonomie solcher collegial formations öffentlich zu legitimieren, politisch zu garantieren und rechtlich abzusichern. Über die historisch verwirklichten Autonomiegarantien für religiöse Sphären, Tarifpartner, freie Vereinigungen hinaus, sollen diese Garantien auch gelten für

"deliberative bodies within modern civil societies as well as professional associations and sites of professionals' practice within corporations, universities, hospitals, artistic networks, and elsewhere." ${ }^{28}$

Diese Theorie des societal constitutionalism hatte ihre Vorläufer in den Ideen zum private government in den USA und zur Mitbestimmung und anderen Formen der Demokratisierung gesellschaftlicher Teilbereiche in Europa, die speziell formale Organisationen unter Konstitutionalisierungsdruck setzten. ${ }^{29}$ Heute kann sie unmittelbar an neuere post-rawlsianische Ansätze der deliberativen Demokratietheorie anschließen, die über politische Akteure hinausgehend, Demokratiepotentiale in gesellschaftlichen Institutionen im nationalen und internationalen Rahmen zu identifizieren sucht und normativ-institutionelle Konsequenzen zieht. ${ }^{30}$ Das Wichtige daran ist, daß deliberative Demokratisierung nicht auf politische Institutionen beschränkt gesehen, sondern explizit in ihrer Ausdehnung auf gesellschaftliche Akteure im nationalen und internationalen Kontext beobachtet wird. ${ }^{31}$ Wichtiger noch erscheinen die Parallelen zur systemsoziologischen Verfassungstheorie, die eine ganz ähnliche Entwicklungslogik von systemischer Expansion und ihrer Beschränkung aufdeckt. In systemtheoretischer Sicht erschöpft sich die historische Rolle der Verfassung, insbesondere ihrer Grundrechte, nicht darin, staatliche Organisation zu normieren und individuelle Rechtspositionen zu schützen, sondern sie besteht primär darin, die Vielfalt gesellschaftlicher Differenzierung gegen Überwältigungstendenzen abzusichern. ${ }^{32}$ Historisch gesehen entstehen Verfassungen im

\footnotetext{
${ }^{25}$ Sciulli (1992), Anm. 24, 56.

26 S c i u lli, ibid., 81.

$27 \mathrm{~S} \mathrm{ci} \mathrm{ulli,} \mathrm{ibid.,} 80$.

${ }^{28} \mathrm{~S}$ c i u ll i , ibid., 208.
}

29 S e I z n i c k, Philip (1992) The Moral Commonwealth: Social Theory and the Promise of Community. Berkeley: University of California Press, 229 ff.; S e I z n i c k, Philip (1969) Law, Society and Industrial Justice. New York: Russell Sage; H a b e r m a s , Jürgen (1962) Strukturwandel der Öffentlichkeit. Neuwied: Luchterhand (5. Aufl. 1996. Frankfurt: Suhrkamp).

${ }^{30}$ D o rf, Michael C. und S a b e I, Charles F. (2003, forthcoming) A Constitution of Democratic Experimentalism. Cambridge (Mass.): Harvard University Press; C o h e n, Joshua und S a b e I, Charles (1997) "Directly-Deliberative Polyarchy" European Law Journal 3, 313-342.

${ }^{31}$ Gerstenberg, Oliver und Sabel, Charles F. (2002) "Directly Deliberative Polyarchy: An Institutional Ideal for Europe?", 289-341; C o h e n , Joshua und R o g e r s , Joel (1998) "Can Egalitarianism Survive Internationalization?" In: Streeck, Wolfgang (Hg.), Internationale Wirtschaft, nationale Demokratie: Herausforderungen für die Demokratietheorie. Frankfurt/M: Campus,175-193.

32 Zum systemtheoretischen Ansatz der Grundrechte als Institution, L u h m a n n, Niklas (1965) Grundrechte als Institution: Ein Beitrag zur politischen Soziologie. Berlin: Duncker \& Humblot. Zu seiner Ausarbeitung in verschiedenen 
Gegenzug zu der für moderne Gesellschaften typischen Emergenz autonomer Handlungssphären. Sobald im politischen System expansionistische Tendenzen aufkommen, die den Proze $B$ sozialer Differenzierung selbst zu ruinieren drohen, kommt es zu gesellschaftlichen Konflikten, als deren Folge Grundrechte als soziale Gegeninstitutionen der Verfassung gerade dort institutionalisiert wurden, wo die soziale Differenzierung von den ihr inhärenten Tendenzen zur Selbstzerstörung bedroht war. Individuelle Konflikte zwischen Privatbürger und Verwaltungsbürokratie dienen zugleich der rechtlich institutionalisierten Absicherung einer Selbstbeschränkung der Politik.

Daraus ergibt sich eine generelle Funktionsbestimmung von Verfassungen im Prozeß der Modernisierung. Polany is berühmte Doppelbewegung von Marktdurchsetzung und Errichten des Schutzmantel kultureller Institutionen findet hier ihre Generalisierung, sofern man die entsprechende Entwicklungsdynamik auch anderer expansiver Sozialsysteme einbezieht. ${ }^{33} \mathrm{Im}$ Prozeß der Konstitutionalisierung geht es darum, das Potential hochspezialisierter Dynamiken durch ihre gesellschaftliche Institutionalisierung freizusetzen. Zugleich aber geht es darum, Selbstbeschränkungsmechanismen gegen ihre gesellschaftsweite Expansion, die sich letztlich gegen die funktionale Differenzierung selbst richtet, zu institutionalisieren. Solche Expansionstendenzen manifestieren sich in historisch sehr unterschiedlichen Konstellationen, früher hauptsächlich in der Politik, heute eher in der Wirtschaft, in der Wissenschaft, der Technologie, und anderen gesellschaftlichen Sektoren. Die Stärkung der Autonomie von Handlungssphären als Gegenbewegung gegen Überwältigungstendenzen scheint der generelle Reaktionsmechanismus zu sein, der in den politischen Verfassungen der Tradition ebenso wie in den emergierenden Zivilverfassungen am Werke ist. War es die zentrale Aufgabe politischer Verfassungen, neben der Organisation des Staatswesens die Autonomie anderer Handlungssphären gerade vor ihrer politischen Instrumentalisierung zu bewahren, so dürfte es in den heutigen Zivilverfassungen darum gehen, gegenüber dem dominanten gesellschaftlichen Rationalisierungstrend die Artikulationschancen sogenannter nicht-rationaler Handlungslogiken dadurch zu sichern, daß Autonomieräume für gesellschaftliche Reflexion in langwierigen Konflikten erkämpft und institutionell garantiert werden. ${ }^{34}$

Aber müßte dies nicht zur vornehmsten Aufgabe gerade einer genuin politischen Verfassung der Weltgesellschaft werden? Dies tiefsitzende Vorurteil dürfte nur schwer auszuräumen sein. Doch zwingen Gewichtsverschiebungen zwischen Politik und anderen gesellschaftlichen Sektoren der Globalisierung dazu, eine weitere einschneidende Änderung von Konstitutionalisierungsprozessen und ihren Trägern ins Auge zu fassen.

\section{Diagnose II: Polyzentrische Globalisierung}

Die Entstehung der Weltgesellschaft findet nicht unter der Führung der internationalen Politik statt, sondern wird von dieser - siehe Globalisierung des Terrorismus - allenfalls reaktiv begleitet. Sie kann auch nicht mit der globalen Vernetzung der Ökonomie gleichgesetzt werden, auf deren Konvulsionen alle anderen Lebensbereiche nur reagieren. Vielmehr ist Globalisierung

Kontexten Ladeur, Karl-Heinz (1999) "Helmut Ridders Konzeption der Meinungs- und Pressefreiheit in der Demokratie" Kritische Justiz 32, 281-300; G r aber, Christoph und Te ubner, Gunther (1998) "Art and Money: Constitutional Rights in the Private Sphere" Oxford Journal of Legal Studies 18, 61-74; Grim m, Dieter (1987) "Grundrechte und Privatrecht in der bürgerlichen Sozialordnung" In: ders. (Hg.), Recht und Staat in der bürgerlichen Gesellschaft. Frankfurt: Suhrkamp,192-211; W ill k e, Helmut (1975) Stand und Kritik der neueren Grundrechtstheorie: Schritte zu einer normativen Systemtheorie. Berlin: Duncker \& Humblot.

${ }^{33}$ P o I a n y i, Karl (1995) The Great Transformation: Politische und Ökonomische Ursprünge von Gesellschaften und Wirtschaftssystemen. Frankfurt: Suhrkamp; eine entsprechende Interpretation des Wirtschaftsrechts als Kollisionsrecht findet sich bei Amstutz, Marc (2001) Evolutorisches Wirtschaftsrecht: Vorstudien zum Recht und seiner Methode in den Diskurskollisionen der Marktgesellschaft. Baden-Baden: Nomos.

${ }^{34} \mathrm{Zu}$ einer solchen Sicht der Konstitutionalisierung des Privatrechts T e u b n e r, Gunther (2000b) "Neo-Spontanes Recht und duale Sozialverfassung in der Weltgesellschaft" In: Zur Autonomie des Individuums. Liber Amicorum Spiros Simitis Baden-Baden: Nomos,169-214; d e r s . (2000a) "Ein Fall von struktureller Korruption? Die Familienbürgschaft in der Kollision unverträglicher Handlungslogiken" Kritische Vierteljahresschrift für Gesetzgebung und Rechtswissenschaften, 388-404; ders. (1999) "Nach der Privatisierung: Diskurskonflikte im Privatrecht" In: MeierSchatz, Christian (Hg.), Die Zukunft des Rechts. Basel: Helbig \& Lichtenhahn,128-161; T e u b n e r, Gunther (1998) "Vertragswelten: Das Recht in der Fragmentierung von Private Governance Regimes" Rechtshistorisches Journal 17, 234-265. Weiterführende Analysen C a Il i e s s, Gralf-Peter (2002) "Reflexive Transnational Law: The Privatisation of Civil Law and the Civilisation of Private Law" Zeitschrift für Rechtssoziologie 24, 185-216; Z u m b a n s e n, Peer (2002) "The Privatization of Corporate Law? Corporate Governance Codes and Commercial Self-Regulation" Juridicum 3 ,3240. 
ein polyzentrischer Prozeß, in dem unterschiedliche Lebensbereiche ihre regionalen Schranken durchbrechen und je für sich autonome Globalsektoren konstituieren. ${ }^{35}$ Sie ist ein

"multidimensional phenomenon involving diverse domains of activity and interaction including the economic, political, technological, military, legal, cultural, and environmental. Each of these spheres involves different patterns of relations and activity." 36

Resultat ist eine Vielheit von selbständigen global villages, die als autonome Funktionsbereiche je für sich eine weltweite und von außen nicht kontrollierbare Eigendynamik entfalten. Globalisierung bedeutet also nicht einfach Globalkapitalismus, sondern die weltweite Realisierung funktionaler Differenzierung. ${ }^{37}$

Entscheidend für unsere Frage ist nun, daß die Globalisierung des Politischen im Vergleich zu anderen Teilsystemen relativ zurückgeblieben ist und auf absehbare Zeit es wohl auch bleiben wird. Angesichts der notorischen Schwäche der Institutionen der Vereinten Nationen ist Weltpolitik im Kern immer noch internationale Politik, also ein Interaktionssystem von autonomen Nationalstaaten, in das allmählich auch internationale Organisationen hineingezogen werden, ohne daß diese die Staatenwelt ablösen oder auch nur auf den zweiten Rang verweisen könnten. In dieser Asymmetrie von voll globalisierten Teilsystemen der Gesellschaft und bloß internationalisierter Politik ist der oben angesprochenen Konstellation der Boden entzogen, in der die politischen Institutionen mit ihrer Eigenverfassung zugleich auch die Gesamtgesellschaft verfassen könnten. Der Nationalstaat konnte noch in Fortsetzung alter Konzepte einer politischen Gesellschaft, in welcher der Monarch als Spitze der Gesellschaft diese repräsentierte, einigermaßen glaubhaft machen, daß das Teilsystem Politik in seiner Staatsverfassung zugleich die gesamte Nation verfaßte, auch wenn die Brüchigkeit dieser Konstruktion schon deutlich wurde. Das bezeugen immer wieder auftauchende Ideen einer eigenständigen Wirtschaftsverfassung, aber auch anderer Teilbereichsverfassungen ebenso wie Konzepte der originären und nicht bloß staatlich verordneten Grundrechtsgeltung in der Zivilgesellschaft. ${ }^{38}$ Für die Weltgesellschaft aber läßt sich ein solcher Anspruch schlechthin nicht mehr geltend machen.

$\mathrm{Da} \beta$ hingegen in der internationalen Politik und in den internationalen Organisationen im engeren Sinne ein realer Konstitutionalisierungsproze $B$ stattfindet, wie er von vielen Völkerrechtlern beobachtet wird, soll damit nicht bestritten, sondern gerade hervorgehoben werden. ${ }^{39}$ Die Entwicklung weltweit geltender Menschenrechte mit Verbindlichkeit gegenüber nationalstaatlichen Gewalten ist dafür der deutlichste Beleg. In unserer Perspektive entscheidend ist nur, daß es sich um die allmähliche Herausbildung einer weltgesellschaftlichen Teilverfassung, der Konstitutionalisierung der internationalen Politik, handelt, die nicht mehr den Anspruch pars pro toto erheben kann. ${ }^{40}$ Einem politikzentrierten Verfassungsdenken ist damit der Boden entzogen. Fahndet man dann nach anderen weltgesellschaftlichen Verfassungselementen, muß man sie in den Eigenverfassungen der globalen Teilsysteme außerhalb der Politik suchen. Auf eine Verfassung der Weltgesellschaft haben die laufenden Konstitutionalisierungsprozessen der internationalen Politik kein Monopol. Für konstitutionelle Konkurrenz sorgt die Autonomisierung

${ }^{35}$ Die Perspektive einer polyzentrischen Globalisierung wird von ganz unterschiedlichen Theorien vertreten, so der neo-institutionalistischen Theorie einer globalen Kultur Me ye r, John W., B oli, John, Th o m a s, George M. und R a m i r e z, Francisco O. (1997) "World Society and the Nation-State" American Journal of Sociology 103, 144-181; der postmodernen Theorie des Rechtspluralismus, S a n t o s, Boaventura de Sousa (1995) Toward a New Common Sense: Law, Science and Politics in the Paradigmatic Transition. New York: Routledge; systemsoziologischen Theorien der differenzierten Weltgesellschaft, St i c hw e h, Rudolf (2000) Die Weltgesellschaft: Soziologische Analysen. Frankfurt: Suhrkamp; und verschiedenen Versionen einer globalen Zivilgesellschaft, G ü n th e r, Klaus und R a n d e ri a, Shalini (2001) Recht, Kultur und Gesellschaft im Prozeß der Globalisierung. Bad Homburg: Reimers; S h a w, Martin (1998) "Die Repräsentation ferner Konflikte und die globale Zivilgesellschaft" In: Beck, Ulrich (Hg.), Perspektiven der Weltgesellschaft. Frankfurt: Suhrkamp, 221-255.

${ }^{36} \mathrm{Held}$, David (1995) Democracy and the Global Order: From the Modern State to Cosmopolitan Governance. Cambridge: Polity Press, 62.

37 Luhmann, Anm. 15, 220 ff. Weiterführende Analysen Albert, Mathias (2002) Zur Politik der Weltgesellschaft: Identität und Recht im Kontext internationaler Vergesellschaftung. Weilerswist: Velbrück; B r u n k h or s t, Hauke (2000) "Ist die Solidarität der Bürgergesellschaft globalisierbar?" In: ders. (Hg.), Globalisierung und Demokratie: Wirtschaft, Recht, Medien. Frankfurt: Suhrkamp, 274-286.

${ }^{38}$ Zur neueren Diskussion um eine Weltwirtschaftsverfassung B e h r e n s, Peter (2000) "Weltwirtschaftsverfassung" Jahrbuch für Neue Politische Ökonomie 19, 5-27; zum konstitutionellen Pluralismus W a I k e r, Anm.11.

39 Dazu neuerdings umfassend Fischer-Lescano, Andreas (2002a) Globalverfassung: Die Geltungsbegründung der Menschenrechte im postmodernen ius gentium. Frankfurt: Juristische Dissertation, der von einer strukturellen Kopplung von globalem Recht und globaler governance spricht; vgl. auch die Analyse von W a I k e $\mathrm{r}$, Anm.11.

${ }^{40}$ So mit wünschenswerter Deutlichkeit F i s c h e r - L e s c a n o (2002a), Anm. 39, Kap. 5 und 6. 
von globalen Teilverfassungen und ihre Vernetzung mit anderen globalen und nationalen Konstitutionalisierungen. ${ }^{41}$

\section{Diagnose III: Creeping Constitutionalism}

Wenn es demnach zutrifft, daß die internationale Politik allenfalls ihre eigene Konstitutionalisierung betreiben kann, nicht aber die der gesamten Weltgesellschaft, wenn weiterhin richtig ist, daß angesichts des evolutionären Drift globaler Rationalisierungsprozesse die normative Notwendigkeit besteht, Autonomieräume der Reflexion zu garantieren, so stellt sich doch die weitere Frage, ob die globalen Gesellschaftssektoren überhaupt das Potential einer Eigenverfassung besitzen. ${ }^{42}$

Hier gilt es, einen wichtigen Zusammenhang zwischen Juridifizierung und Konstitutionalisierung herauszustellen. Mit Notwendigkeit enthält jeder Prozeß der Verrechtlichung zugleich latente konstitutionelle Normierungen. In den Worten eines Verfassungsrechtlers:

"Nicht jedes Gemeinwesen hat eine geschriebene Verfassung, aber jedes Gemeinwesen hat

Verfassungsrecht. Dieses Verfassungsrecht muß mindestens die Hauptakteure konstituieren und gewisse Verfahrensregeln enthalten. Theoretisch könnte eine Verfassung sich damit begnügen, ein Organ der Rechtssetzung einzusetzen und zu regeln, wie dieses Organ Gesetze beschließt." ${ }^{\prime 3}$

Letztlich geht es hier um die konstitutionelle Qualität jeglicher Emergenz von Rechtsnormen, also um die schwierigen Fragen der nicht begründbaren Fundamente des Rechts, die von den großen Rechtstheorien unserer Zeit umkreist werden. Die sich hier stellenden Sachprobleme heißen: Selbstbegründung des Rechts, dabei auftauchende Paradoxien, die den Rechtsprozeß blockieren, deren wirksame, aber immer problematisch bleibende Auflösung durch autologische Qualitäten der Konstitutionalisierung. Diese Qualitäten werden von Kels e n im Verhältnis der Grundnorm zur höchsten Verfassungsnorm, von Hart in der Theorie der sekundären Regeln und der ultimate rule of recognition, von Luhman $\mathrm{n}$ in der Beziehung von Rechtsparadox und Verfassung und von Derridas Ideen über die paradoxe Rechtsgründungsgewalt in stets neuen Varianten durchgespielt. ${ }^{44}$ Immer wieder geht es darum, den paradoxen Prozeß zu verstehen, in dem jede Rechtsbildung immer schon rudimentäre Elemente ihrer Eigenverfassung voraussetzt und diese zugleich erst in ihrem Vollzug konstituiert.

In unserem Zusammenhang kommt es nun darauf an, daß sich das problematische Verhältnis von Verrechtlichung und Konstitutionalisierung heute nicht mehr nur auf politische Gemeinwesen begrenzen läßt. Grotius berühmter Satz "ubi societas ibi ius" ist unter den Bedingungen funktionaler Differenzierung des Globus so umzuformulieren, daß wo immer sich autonome Gesellschaftssektoren entwickeln, zugleich eigenständige Mechanismen der Rechtsproduktion herausgebildet werden, die in relativer Distanz zur Politik stehen. Rechtssetzung findet auch außerhalb der klassischen völkerrechtlichen Quellen statt, in Verträgen zwischen global players, in privater Marktregulierung durch multinationale Unternehmen, internen Regelsetzungen internationaler Organisationen, interorganisationalen Verhandlungssystemen, weltweiten Standardisierungsprozessen, die sich teils in Märkten teils in Verhandlungsprozessen von Organisationenabspielen. ${ }^{45}$

"Regulations and norms are produced not only by negotiations between states, but also by new semi-public, quasi-private or private actors which respond to the needs of a global market. In-between

\footnotetext{
${ }^{41}$ W alter, Anm.11, 173; W alker, Anm.11.

${ }^{42}$ Eine eindringliche Analyse des internationalen Rechtspluralismus in systemtheoretischer Perspektive findet sich bei O e t e r, Stefan (2001) "International Law and General Systems Theory" German Yearbook of International Law 44, 72-95.

${ }^{43}$ Uerpmann, Anm. 9, 566. Ebenso Tomuschat, Anm. 9, 217.

${ }^{44}$ Kels e n, Hans (1946) General Theory of Law and State. Cambridge Mass.: Harvard University Press, 116; H a r t , Herbert L.A. (1961) The Concept of Law. Oxford: Clarendon, $77 \mathrm{ff}$. ; L u h m a n n , Niklas (1990) "Verfassung als evolutionäre Errungenschaft" Rechtshistorisches Journal 9, 176-220, 184; D e r r i d a, Jacques (2000) "Otobiographien: Die Lehre Nietzsches und die Politik des Eigennamens" In: ders. (Hg.), Nietzsche - Politik des Eigennamens: Wie man abschafft, wovon man spricht. Berlin: Merve,7-63, 9 ff.; Derrida, Jacques (1991) Gesetzeskraft: Der "mystische Grund der Autorität". Frankfurt: Suhrkamp.

${ }^{45}$ Albert, Anm. 37; Günther und Randeria, Anm. 35; Robe, Jean-Philippe (1997) "Multinational Enterprises: The Constitution of a Pluralistic Legal Order" In: Teubner, Gunther (Hg.), Global Law Without A State. Aldershot: Dartmouth Gower, 45-77; B i a n c h i , Andrea (1997) "Globalization of Human Rights: The Role of Non State Actors" In: Teubner, Gunther (Hg.), Global Law Without A State. Aldershot: Dartmouth Gower,179-212; S a n t o s, Anm. 35.
} 
states and private entities, self-regulating authorities have multiplied, blurring the distinction between the public sphere of sovereignty and the private domain of particular interests". 46

Und Rechtsnormproduktion aus Konfliktlösung vollzieht sich nicht nur innerhalb von nationalen und internationalen Gerichten, sondern auch innerhalb von nichtpolitischen gesellschaftlichen Konfliktlösungsinstanzen, internationalen Organisationen, Schiedsgerichten, Mediationsinstanzen, Ethikkommissionen, Vertragsregimes. Wenn es richtig ist, daß die dominanten Rechtsquellen sich nun an den Peripherien des Rechts, an den Grenzen zu anderen Sektoren der Weltgesellschaft, und nicht mehr nur in den bisherigen Zentren der Rechtsbildung nationalen Parlamenten, und zwischenstaatlichen Vereinbarungen - befinden, dann heißt dies zugleich, daß dort immer auch Normen konstitutioneller Qualität produziert werden.

Die neuen Phänomene einer globalen Verrechtlichung implizieren also die Möglichkeit, daß sich Konstitutionalisierungsprozesse auch außerhalb staatlicher und politischer Institutionen abspielen. ${ }^{47}$ Das bedeutet freilich nicht, daß nun jeder Gesellschaftssektor seine konstitutionellen Normen ausschließlich in Eigenregie produziert. Ebenso wie die globale Verrechtlichung gesellschaftlicher Teilbereiche stets ein Mischungsverhältnis von autonomer und heteronomer Rechtsbildung aufweist ${ }^{48}$ ist auch die Herausbildung von globalen Zivilverfassungen ein Prozeß, an dem externe und interne Faktoren zusammenwirken. Stets ist das Rechtssystem beteiligt, denn diese Prozesse finden im gesellschaftlichen Subsystem und zugleich an der Peripherie des Rechts statt. Und in mehr oder minder großem Ausmaß spielt die internationale Politik bei der Herausbildung von globalen Subverfassungen eine Rolle, indem sie diese mit politischen Verfassungsinterventionen irritiert. Wie im einzelnen das Mischungsverhältnis zwischen externer politischer und autonomer gesellschaftlicher Konstitutionalisierung ausgestaltet ist, ist letztlich eine empirische Frage, die von einmaligen historischen Konstellationen abhängt. Doch insofern autonomes Weltrecht sich auf eigene Ressourcen stützt und internationale Organisationen, Nichtregierungsorganisationen, Medien, multinationale Unternehmen, globale Anwaltspraktiken, globale Funds, globale Verbände, globale Schiedsgerichte den globalen Rechtsbildungsprozeß vorantreiben, ist autonome Rechtssetzung auch an dem Formierungsprozeß ihrer sektorialen Verfassung maßgeblich beteiligt.

Schließlich ist hier ein merkwürdiges Latenzphänomen zu beobachten. Elemente der Zivilverfassungen sind nicht in einem big bang, in einem spektakulären revolutionären Akt der Konstituente nach amerikanischem oder französischem Vorbild entstanden. Ebensowenig kennen die globalen Netzwerke der Wirtschaft, der Forschung, des Gesundheitswesens, der Erziehung, der Professionen den einen großen Urtext, der als Kodifikation in einem Verfassungsdokument niedergelegt wäre. Eher bilden sich Zivilverfassungen in untergründigen evolutionären Prozessen von langer Dauer heraus, in denen sich im Zuge der Verrechtlichung von Gesellschaftssektoren auch konstitutionelle Normen inkrementell entwickeln, wobei sie im Ensemble der Rechtsnormen eingebettet bleiben. Im Nationalstaat blendet der Glanz der politischen Verfassung so sehr, daß die Eigenverfassungen der Zivilsektoren nicht sichtbar sind oder allenfalls als Resultat politischer Verfassungsgebung aufscheinen. Aber auch im globalen Maßstab sind sie ebenso nur latent vorhanden, auf merkwürdige Weise invisibilisiert.

Wie häufig läßt sich auch hier manches am Sonderfall England lernen. Wenn auch auf dem Kontinent gern das Vorurteil gepflegt wird, England habe keine Verfassung oder sei verfassungsrechtlich unterentwickelt, so sind doch im Gefolge der Analysen von Dice y die konstitutionellen Qualitäten des britischen Gemeinwesens und des common law immer wieder klar herausgearbeitet worden. ${ }^{49}$ Ihre inhaltlichen Qualitäten in Bezug auf Staatsorganisation und Grundrechte, insbesondere deren Schutzintensität, können jedem Vergleich mit kontinentalen Verfassungen standhalten. Auf die soziale Institutionalisierung einer Verfassung kommt es an und nicht auf die formelle Existenz einer konstituierenden Versammlung, einer Verfassungsurkunde, von Normen explizit verfassungsrechtlicher Qualität oder eines auf Verfassungsfragen spezialisierten Gerichts. Mutatis mutandis gilt dies auch für die

${ }^{46}$ Gué he n no , Jean-Marie (1998) "From Territorial Communities to Communities of Choice: Implications for Democracy" In: Streeck, Wolfgang (Hg.), Internationale Wirtschaft, nationale Demokratie: Herausforderungen für die Demokratietheorie. Frankfurt/M: Campus,137-150,141

${ }^{47} \mathrm{~W}$ a lt e r, Anm. 11, 194; W alk e r, Anm. 11

48 Dies muß eigens hervorgehoben werden, da sonst rechtspluralistische Ansätze allzuleicht (und allzu gern) mißverstanden werden, als schlössen sie zugunsten autonomer Rechtsbildung externe politische Rechtsinterventionen aus. Globales Wirtschaftsrecht etwa wird in rechtspluralistischer Sicht ausdrücklich als empirisch variables Mischungsverhältnis aus ökonomischer und politischer Rechtsbildung gesehen. Siehe T e u b n e r, Gunther (1996) "Globale Bukowina: Zur Emergenz eines transnationalen Rechtspluralismus" Rechtshistorisches Journal, $255-290$.

${ }^{49} \mathrm{D}$ i c e y , Albert (1964) An Introduction to the Study of the Law of the Constitution. London: MacMillan. 
Zivilverfassungen der Weltgesellschaft. Die Latenz von konstitutionellen Elementen zu aktualisieren, brächte es dann mit sich, daß man faktische Abläufe der Konstitutionalisierung normativ zu reflektieren und sie in ihrer Richtung zu beeinflussen, in der Lage ist.

\section{Konturen der Zivilverfassungen. Beispiel: Digitalverfassung}

Welche Merkmale müssen vorliegen, damit man von der Existenz konstitutioneller Elemente in den verschiedenen globalen Sektoren sprechen kann $?^{50}$ In der Tat, die politischen Verfassungen der nationalstaatlichen Tradition können als das große historische Vorbild auch für Zivilverfassungen dienen. Hier steht ein Fundus von historischen Erfahrungen, an Prozeduren, Begriffen, Prinzipien, Normen zur Analogie für die heutige Lage zur Verfügung. Jedoch sind die Analogien mit äußerster Vorsicht zu ziehen, da man allzu leicht kurzschlüssig transponiert und die Besonderheiten der globalisierten Gesellschaftssektoren verpaßt.

Das gilt schon für das Ausmaß der Konstitutionalisierung. Dies ist hoch variabel. Nirgendwo steht geschrieben, daß die umfassende Konstitutionalisierung, die den gesamten Politikprozeß mit einem dichten Gewebe an Verfassungsnormen überzieht, sich in den Verfassungen gesellschaftlicher Teilbereiche - man denke nur an Forschung oder Kunst - wiederholen müßte. Immer geht es, wie schon eingangs gesagt, um sorgfältige Generalisierung und Respezifizierung des Verfassungsphänomens zugleich. Generalisierung bedeutet, den Verfassungsbegriff von bestimmten Eigenheiten des politischen Systems und besonders des Staates abzulösen, was aber angesichts der engen Verflechtung konstitutioneller und politischer Momente außerordentlich delikat ist. Respezifizierung ist dann eine nicht minder delikate Angelegenheit, da die Besonderheiten der subsystemspezfischen Operationen, Strukturen, Medien, Codes und Programme ein weitgehendes Umdenken verfassungsrechtlicher Institutionen erfordern.

Um dies an einem Problem der globalen Wissenschaftsverfassung zu verdeutlichen: Wie lassen sich konstitutionelle Abstützungen der Forschungsfreiheit gegenüber ökonomischen Einflüssen denken? Ein zu enger Analogieschluß von politischer Macht auf wirtschaftliche Macht würde weder das grundrechtsgefährdende Medium noch die adäquate Sanktionierung ausreichend generalisieren und respezifizieren. Das Kriterium kann nicht einfach, wie politikinspirierte Überlegungen immer wieder nahelegen, die soziale Machtposition wirtschaftlicher Akteure sein, sondern erst die Gefährdung durch das spezifische kommunikative Medium des jeweiligen expansiven sozialen Systems. Forschungsfreiheit wird entsprechend nicht nur durch die überwältigenden Machtstrukturen von Mega-Unternehmen gefährdet, gegen die machtlose Individuen protestieren. Vielmehr stammen die neuen Gefahren besonders aus der strukturellen Korruption durch das Geldmedium. Abhängigkeit der Forschung vom Markt bezeichnet die neuen Verführungssituationen durch wirtschaftliche Anreize, die sich dann freilich auch nicht durch verfassungsrechtliche Garantien von subjektiven Grundrechten als geschützter Autonomieräume konterkarieren lassen. In einer solchen Generalisierung/Respezifikation der konstitutionellen Problemlage und der Reaktionsmöglichkeiten wird deutlich, daß es eine sehr viel effektivere Verfassungsgarantie wäre, wenn man die monetären Abhängigkeitsquellen der Forschung vervielfachte, um aus den vielen Abhängigkeiten eine neue Unabhängigkeit zu schaffen. Teufel mit Beelzebub austreiben! Könnte die Wissenschaftsverfassung eine Vielheit von unterschiedlichen gegeneinander konkurrierenden Finanzquellen für die Forschung nicht nur normieren, sondern auch faktisch garantieren, so hätte dies Auswirkungen auf die Autonomie der Wissenschaft, die den Vergleich mit der Wirkung traditioneller subjektiver Rechte gegenüber politischen Interferenzen nicht zu scheuen brauchte. ${ }^{51}$

\section{Erstes Merkmal: Strukturelle Kopplung Teilsystem - Recht}

Zivilverfassungen sind weder bloße Rechtstexte, noch geht es nur um die faktische Verfaßtheit von Sozialordnungen. ${ }^{52}$ Von Elementen einer Zivilverfassung im strengen Sinne sollte man erst dann sprechen, wenn ein voraussetzungsreiches Zusammenspiel von autonomen Sozialprozessen und autonomen Rechtsprozessen zustandekommt, in systemtheoretischer Sprache, wenn auf Dauer gestellte strukturelle Kopplungen von teilbereichsspezifischen

\footnotetext{
${ }^{50}$ Eindringliche Analysen bei W a lk e r, Anm. 11, und W a It e r, Anm. 11.

51 Zur Freiheit der Wissenschaft in dieser Sicht Ke a ly, Terence (1997) "It's Us Against Them" Guardian May, 7; zur Freiheit der Kunst, G r a b e r und T e u b n e r, Anm. 32.

${ }^{52}$ Richtig Behrens, Anm. 38 , zur Wirtschaftsverfassung.
} 
Ordnungsmustern und Rechtsnormen eingerichtet sind. ${ }^{53}$ Dann erst findet man die merkwürdige Verdoppelung des Verfassungsphänomens vor, die für strukturelle Kopplung kennzeichnend ist und die das verbreitete Verständnis ausschließt, das von der Verschmelzung in einem einheitlichen Verfassungsbegriff, der Rechtsordnung und Sozialordnung übergreift, ausgeht. Verfassung ist immer erst die Verknüpfung zweier realer Prozesse: Aus der Sicht des Rechts ist sie Rechtsnormenproduktion, die eigentümlich mit Grundstrukturen des Sozialsystems verflochten ist; aus der Sicht des verfaßten Sozialsystems ist sie Erzeugung von Grundstrukturen der Sozialordnung, die zugleich das Recht informieren und ihrerseits vom Recht normiert werden. ${ }^{54}$ Der Witz struktureller Kopplung ist, daß sich dadurch beide - der Rechtsproze $\beta$ und der Sozialproze $B$ - in ihren Beeinflussungsmöglichkeiten wechselseitig beschränken. Es werden Überwältigungen der einen Ordnung durch die andere blockiert, ihre jeweiligen Autonomien ermöglicht und wechselseitige Irritationen auf eng begrenzte und offen institutionalisierte Einflußwege konzentriert.

Damit reagiert die Verfassung, sofern sie in dieser Weise als Kopplung zweier Sinnsphären institutionalisiert ist, auf ein Problem, das bei jeder autonomen gesellschaftlichen Normbildung auftaucht: auf das Problem struktureller Korruption. So hat die heute heiß umkämpfte Frage, ob und wie und durch welche Akteure das Internet reguliert werden soll, genau hiermit zu tun. ${ }^{55}$ Nationale Regulierung scheitert an Implementationsproblemen, die der transnationale Charakter der digitalen Kommunikation aufwirft. Die heute von allen Gutmenschen erwünschte InternetRegulierung durch völkerrechtlich legitimierte internationale Rechtssetzung wiederum droht an den Schwierigkeiten der zwischenstaatlichen Konsensbildung zu scheitern. Das schließt natürlich nicht aus, daß beides weiterhin versucht und teilweise auch mit Erfolg verwirklicht wird. Doch bringen die faktischen Schwierigkeiten beider Regulierungsformen es mit sich, daß Selbstregulierung des Internet dramatisch aufgewertet wird. Deshalb sprechen Beobachter der Internetregulierung von einem "trend toward self-regulation". ${ }^{56}$ Das selbstgemachte Recht des Internet profitiert nicht nur von den Schwierigkeiten der beiden anderen Regulierungsformen, sondern besonders von den technischen Vorteilen, welche die Architektur des Code für eine hocheffiziente Regulierung bietet. Dank elektronischer Zwangsmittel kann sie weitgehend auf erwartungsgesteuerte Regulierung verzichten, ist aber ihrerseits von Meta-Rechtsnormen gesteuert. ${ }^{57}$ Der Entwicklungstrend geht also deutlich in Richtung hybrider Regulierung: "a private, transnational, self-executing and mandatory mechanism under public review". ${ }^{8}$ In der Mischung von verschiedenen Regulierungsformen spielt eine autonome lex electronica, in Parallele zur autonomen lex mercatoria des autonomen Wirtschaftsrechts, eine maßgebliche Rolle. ${ }^{59}$ Die Schiedsgerichte des ICANN, die aufgrund der autonomen anationalen Rechtsnorm des $\S 12 a$ der ICANN policy über die domain-Vergabe rechtsverbindlich und mit elektronischer Zwangsvollstreckung entscheiden, sind Teil einer solchen autonomen Rechtsbildung im Internet. Und in genauer Parallele zum globalen Wirtschaftsrecht entsteht mit der lex electronica das

${ }^{53}$ Zur strukturellen Kopplung des Rechts mit anderen Sozialsystemen T e u bn e r, Gunther (2002) "Idiosyncratic Production Regimes: Co-Evolution of Economic and Legal Institutions in the Varieties of Capitalism" In: Ziman, John (Hg.), The Evolution of Cultural Entities: Proceedings of the British Academy. Oxford: Oxford University Press, 161-181; Luh m a n n, Anm. 4, $440 \mathrm{ff}$.

${ }_{55}^{54} \mathrm{~L} \mathrm{u} \mathrm{h} \mathrm{m} \mathrm{a} \mathrm{n} \mathrm{n,} \mathrm{Anm.} \mathrm{44,} \mathrm{für} \mathrm{den} \mathrm{Fall} \mathrm{der} \mathrm{Kopplung} \mathrm{Politik/Recht.}$

${ }^{55} \mathrm{Holznagel}$, Bernd und Werle, Raymund (2002) "Sectors and Strategies of Global Communications Regulation" Zeitschrift für Rechtssoziologie 23, 3-23; Committee to Study Global Networks and Local Values, Computer Science and Telecommunications Board, National Research Council (2001) Global Networks and Local Values: $A$ Comparative Look at Germany and the United States. Washington, DC: National Academy Press; Le s s i g, Anm. 18, und J o hns on und Post, Anm. 18.

${ }^{56} \mathrm{Holzn}$ agel und We rle, Anm. 55, 18; Golds mith, Jack (2000) "The Internet, Conflicts of Regulation and International Harmonization" In: Engel, Christoph (Hg.), Governance of Global Networks in the Light of Differing Local Values. Baden-Baden: Nomos.

${ }^{57}$ Le s sig, Anm. 18, $43 \mathrm{ff}$.

58 Lehmkuhl, Dirk (2002) "The Resolution of Domain Names vs. Trademark Conflicts: A Case Study on Regulation Beyond the Nation State, and Related Problems" Zeitschrift für Rechtssoziologie 23, 61-78, 62, $64 \mathrm{ff}$. Dazu auch F a r r e II, Henry (2002) "Hybrid Institutions and the Law: Outlaw Arrangements or Interface Solutions" Zeitschrift für Rechtssoziologie 23, 25-40; P e rritt, Henry H. (2000) "Hybrid International Institutions for Regulating Electronic Commerce and Political Discourse on the Internet" Multimedia und Recht 7/Beilage, 1-3.

59 Die Gemeinsamkeiten und Unterschiede von lex mercatoria und lex digitalis werden deutlich herausgearbeitet von C alli i s s, Anm. 34. Zur Autonomie der lex digitalis B e r n s t o r f f , Jochen von (2003) "Internet 'Law': Legitimacy and Legal Structures of ICANN" In: Joerges, Christian, Sand, Inger und Teubner, Gunther (Hg.), Constitutionalism and Transnational Governance. Baden-Baden: Nomos (im Erscheinen); L e h m k u h l, Anm. 58; T h o r n b u r g, Elisabeth (2000) "Going Private: Technology, Due Process and Internet Dispute Resolution" University of California Davis Law Review 34, 151-220. 
Problem der strukturellen Korruption, also des massiven ungefilterten Einflusses von "privaten" Interessen auf den Rechtsbildungsprozeß . Hier stellt sich die Verfassungsfrage. ${ }^{60}$

Um Chancen und Grenzen einer Digitalverfassung realistisch einzuschätzen, sollte man politische Verfassungen, die auf das Problem struktureller Korruption des Rechts durch die Politik reagiert haben, als Vorbild benutzen. ${ }^{61}$ Auf die diffuse Abhängigkeit des vorneuzeitlichen Rechts von politischen Pressionen, politischem Terror, von Positionen sozialer und wirtschaftlicher Macht gaben politische Einrichtungen struktureller Kopplung die Doppelantwort, die Korruption natürlich nicht beseitigen, aber doch eindämmen konnte: Illegalisierung korrumpierender Einflüsse einerseits und Zunahme von legitimer Irritabilität andererseits. Für parallelgelagerte Probleme der Korruption des Rechts durch die Wirtschaft gab dann nicht die politische Verfassung entsprechende Antworten, sondern die Wirtschaftsverfassung selbst übernahm mit den privatrechtlichen Institutionen von Eigentum und Vertrag eine ähnliche Funktion. Die Käuflichkeit des Rechtsmechanismus selbst wurde strikt ausgeschlossen und die wirtschaftlichen Irritationen des Rechts wurden über den Vertragsmechanismus kanalisiert. Zugleich wurde es dadurch möglich, deren Letztkontrolle dem Recht und der Politik vorzubehalten. ${ }^{62}$ Eine realistische Antwort auf Probleme struktureller Korruption des cyber law dürfte entsprechend auch nur die Eigenverfassung des Internet geben, sofern es gelingt, eine funktionierende strukturelle Kopplung zwischen digitalen Kommunikationsstrukturen und fundamentalen Rechtsnormen herzustellen. $\mathrm{Ob}$ und inwieweit eine solche Eigenverfassung politisch extern oktroyiert wird, sei es unilateral durch die US-Regierung, sei es durch ein internationales Regime, oder ob sie sich als interner Selbstorganisationsprozeß des Internet durch Institutionen wie ICANN und deren Schiedsgerichte, Standardisierungsorganisationen wie das World Wide Web Consortium oder die Internet Engineering Task Force und digitale Bürgerbewegungen oder in einem hybriden Proze $B$ herausbildet, ist eine ganz andere Frage. ${ }^{63}$ Sie ändert aber nichts an der Notwendigkeit einer eigenständigen Digitalverfassung als einer effektiven strukturellen Kopplung des Rechts an digitale Kommunikation.

\section{Zweites Merkmal: Normhierarchie}

Strukturelle Kopplung von Sozialordnung und Recht ist notwendige Bedingung einer Zivilverfassung, aber sie ist nicht hinreichend. Denn es gibt Myriaden von wechselseitigen Irritationen, die aber selbst nicht Verfassungsqualität annehmen. Zurückgewiesen wird damit ein Begriff von Zivilverfassung in Parallele zu einem Begriff der Wirtschaftsverfassung, der als "G e s a m th e it der für das gesellschaftliche Wirtschaften verbindlichen Rechtsregeln"64 definiert wurde. Zur Rechtsnormqualität und zur strukturellen Kopplung mit der Sozialordnung hinzukommen muß eine spezifische autologische Beziehung, eine Hierarchisierung von Normen höherer verfassungsrechtlicher Qualität und niedriger einfacher Rechtsqualität.

In erster Linie geht es um Selbsterzeugungsregeln des Rechts, um Verfassungsnormen also, die dem paradoxen Erfordernis genügen müssen, die rechtmäßige Erzeugung von Rechtsnormen zu regulieren, zugleich aber auch ihre eigene Erzeugung zu regeln oder stattdessen auf revolutionären Gewaltakt, Sozialvertrag, göttliche Stiftung oder andere Gründungsmythen zu verweisen. Besonders einflußreich ist hier die Konzeption von Herbert $\mathrm{Hart}$ geworden, der von Recht erst dann spricht, wenn die konstitutionelle Differenz von primären Normen (Verhaltenssteuerung) und sekundären Normen (Rechtserzeugung) eingerichtet ist, der sich damit aber auch das Problem einhandelt, einen infiniten Regreß von Metameta...-Normen mit der Willkür einer ultimate rule of recognition abbrechen zu müssen. ${ }^{65}$ Die zivilverfassungsrechtliche Herausforderung besteht hier darin, eigenständige Selbsterzeugungsregeln zu identifizieren, die die Sichtverengungen eines politikzentrierten Rechtserzeugungsbetriebs überwinden. Hatte schon die politische Verfassungstradition Schwierigkeiten mit der Rechtsnormqualität von genuinem Richterrecht, von Völkerrecht, von privaten Verträgen, privaten Organisationsnormen und von Gewohnheitsrecht, weil in diesen Fällen die "offiziellen" sekundären Normen, die in positivierten Verfassungen auf parlamentarische Gesetzgebung verweisen, versagten, so

\footnotetext{
${ }^{60}$ Vgl. die Kritik von LehmkuhI, Anm. 58, 67 ff.; Geist, Michael (2001) "Fair.com? An Examination of the Allegations of Systemic Unfairness in the ICANN UDRP", http://aix1.uottawa.ca/ geist/geistudrp.pdf

${ }^{61} \mathrm{Luhm}$ a n n, Anm. 4, $468 \mathrm{ff}$.

62 L u h m a n n, ibid., $452 \mathrm{ff}$.

${ }^{63}$ Dazu F a r r e II, Anm. 58.

${ }^{64}$ Stre it, Manfred E. (1991) Theorie der Wirtschaftspolitik. Düsseldorf: Werner, 24.

${ }^{65} \mathrm{H}$ a rt, Anm. 44, $77 \mathrm{ff}$.
} 
vervielfältigen sich die Probleme im Falle autonomer Rechtsregimes in den Weiten der Weltgesellschaft. Über diese Fragen gibt es im Falle der lex mercatoria eine dreißigjährige erhitzte Diskussion ${ }^{66}$ im Falle der lex electronica beginnt sie erst allmählich heißzulaufen. ${ }^{67}$ Die Diskussion gewinnt an Hitze, wenn bewußt wird, daß die Sekundärnormen nicht nur die kognitive Frage "Was ist geltendes Recht", sondern auch die normative Frage nach den legitimen Akteuren und Verfahren der Rechtserzeugung beantworten.

Welches sind die sekundären Normen, die den Umschlag der netiquette, also der guten Sitten des Internet (kein spamming etc.) in digitales Gewohnheitsrecht mit universalem Geltungsanspruch definieren? Auf welche verfassungsrechtliche Ermächtigung können sich die Standardorganisationen des Internet stützen, wenn sie die digitalen Kommunikationsregeln verkünden und in der Architektur des Internet auch gleich implementieren? Nach welchen rules of recognition richten sich die privaten Internet-Schiedsgerichte, die über Domain-Streitigkeiten mit Verbindlichkeitsanspruch entscheiden und sie, wenn eine kurze Frist zu Einspruch bei nationalen Gerichten verstrichen ist, gleich mit elektronischer Zwangsvollstreckung durchsetzen? Über welche sekundären Normen regelt sich die Rechtsqualität von click wrap rules, Allgemeinen Geschäftsbedingungen der Internet-Provider und host provider, die wie in unserem harmlosen Rechtsfall über den Zugang zu den Einrichtungen des Internet verbindlich entscheiden? Etatisten machen es sich zu leicht, wenn sie dies alles als Rechtsphantasien überspannter HarvardProfessoren abtun; eine realistische Sicht wird anerkennen, daß im Laufe solcher selbstorganisierter Rechtspraktiken, die wegen der notwendigen Vertextung digitaler Kommunikation immer auch hochformalisiert sind, zugleich auch - jedenfalls implizit konstitutionelle Sekundärnormen entstehen, die das Geltungsparadox eines selbstgemachten Digitalrechts zu überwinden vermögen und über die Rechtsnormqualität von sozialen Normen selektiv entscheiden.

\section{Drittes Merkmal: Inhaltliche Normenkontrolle an Grundrechtsmaßstäben}

Normenhierarchie heißt nicht nur Selbsterzeugung, sondern auch Selbstkontrolle des Rechts. Das Recht selbst deklariert rechtmäßig erlassene Normen als rechtswidrig, wenn sie zu höherrangigen verfassungsrechtlichen Normen inhaltlich im Widerspruch stehen. In hochentwickelten politischen Verfassungen hat dies bekanntlich zur Differenzierung von Verfassungsgerichtsbarkeit/einfacher Gerichtsbarkeit und von Verfassungsnormen/einfachen Rechtsnormen geführt. Wenn sich nun in den unterschiedlichen gesellschaftlichen Teilbereichen solche expliziten Differenzierungen nicht finden lassen, so bedeutet dies nicht, daß hier keine Normenhierarchien bestünden und keine Normenkontrollen stattfänden. Die gerichtlichen Kontrollen von Allgemeinen Geschäftsbedingungen, von privaten Standards, von Normierungenen privater Verbände, von Schiedsgerichtsentscheidungen im nationalen wie im internationalen Bereich sind in der Sache verfassungsrechtliche Kontrollen nicht-legislativen Rechts. Man soll sich von den antiquierten privatrechtlichen Kontrollformeln der "guten Sitten", "Treu und Glauben", die die ordentlichen Gerichte benutzen, nicht darüber täuschen lassen, daß hier materiell über ordre public, also über die Übereinstimmung von "privaten" Normierungen mit Verfassungsmaßstäben, insbesondere den Grundrechten, entschieden wird. Dies wird mit der Drittwirkung von Grundrechten in gesellschaftlichen Bereichen begründet. Jedoch zeigt sich bei näherem Hinsehen, daß die privaten Normen nicht an der politischen Verfassung, sondern an ihrer Eigenverfassung gemessen werden. Immer geht es zugleich um juridische Entfesselung und um Zügelung systemspezifischer Rationalität. Es geht also um die institutionelle Dimension

\footnotetext{
${ }^{66}$ Z u m b a n s e n, Peer (2002) "Piercing the Legal Veil: Commercial Arbitration and Transnational Law", European Law Journal 8, 400-432; B e r g e r, Klaus-Peter (2000) "Understanding International Commercial Arbitration" In: Center of Transnational Law (Hg.), Understanding International Commercial Arbitration. Münster: Quadis; Le h m k u h I, Dirk (2000) "Commercial Arbitration - A Case of Private Transnational Self-Governance?" Preprints aus der Max-PlanckProjektgruppe Recht der Gemeinschaftsgüter; Te ubner, Gunther (1997) "Global Bukowina: Legal Pluralism in the World Society" In: ders. (Hg.), Global Law Without A State. Aldershot: Dartmouth Gower, 3-28; S t e in, Ursula (1995) Lex mercatoria: Realität und Theorie. Frankfurt: Klostermann.

${ }^{67}$ Le h m kuhl, Anm. 58, 67 ff.; B rous s e a u, Eric (2001) "Internet Regulation: Does Self-Regulation Require an Institutional Framework?" In: http://www.isnie.org/ISNIE01/Papers01/brousseau.pdf; F r o o m k i n , A. Michael (2000) "Semi-private International Rulemaking: Lessons Learned from the WIPO Domain Name Process" In: Mardsen, Christopher (Hg.), Regulating the Global Information Society London: Routledge, 211-232; G e i s t , Anm. 60.
} 
der Grundrechte in "privaten" Gesellschaftsbereichen. ${ }^{68}$ Gesellschaftliche Normierungen in der Peripherie des Rechtssystems werden im Zentrum des Rechts akzeptiert, aber in einem Prozeß gerichtlicher Kontrolle des Rechts werden korrumpierende Elemente, die aus den Rechtsdefizienzen der externen Rechtsquelle stammen, abgewehrt. Zugleich aber erkennt das Recht die Eigenrationalität der externen Rechtssetzungsprozesse an, übersetzt diese in Rechtsnormqualität und bewirkt dadurch inre beträchtliche gesellschaftliche Aufwertung. Die Konsequenz heißt: "setting up an internal human rights standard for the international regime concerned". ${ }^{69}$ Regimespezifische Grundrechtsstandards sind nicht nur wegen der Grundrechtskollisionen zwischen nationalen Verfassungen notwendig, sondern grundsätzlicher noch wegen der Eigenrationalität und Eigendynamik der jeweiligen Regimes.

Im Verhältnis zur Politik hat die nationalstaatliche verfassungsgerichtliche Normenkontrolle das Modell vorgelegt, das im Verhältnis zu anderen Teilsystemen bisher nur rudimentär vorhanden ist. In welcher Hinsicht muß sich das Recht an die Eigenrationalität des anderen Teilsystems lernend anpassen, in welcher Hinsicht müssen rechtskorrumpierende Einflüsse abgewehrt werden? In der Verfassungskontrolle politischer Rechtssetzung sind extensive Kontrolltechniken entwickelt worden, die parteipolitische Entscheidungen relativ neutralisieren, ergebnisorientierte policies als universale Rechtsprinzipien rekonstruieren, politische Entscheidungen in die Rechtsdogmatik nach juristischen Konsistenzkriterien einpassen und im schlimmsten Fall Gesetzgebungsakte als verfassungswidrig deklarieren. Andererseits hat das Verfassungsrecht die Eigenlogik der Politik freigesetzt, indem es das Recht selbst "politisierte": Teleologische Interpretation, Policy-Orientierung, Interessenabwägung, impact assessment und Folgenorientierung sind Indikatoren für eine Anpassung des Rechts an die Rationalität der Politik. $^{70}$

Wo aber liegen die analogen Kombinationen von Freisetzung und Zügelung, die gegenüber nicht-politischen Gesellschaftssektoren herausgebildet werden, wenn hier nicht-legislative Rechtssetzungsmechanismen am Werke sind? Offensichtlich müssen die Kontrollkriterien und die Anpassungsmechanismen der politischen Verfassung durch solche der Eigenverfassung abgelöst werden. Globale technologische Standards bedürfen anderer Rechtskontrollen, anderer Kontrollmaßstäbe, anderer Kontrollverfahren als internationale Allgemeine Geschäftsbedingungen oder globale Verhaltenscodices internationaler professioneller Verbände.

Im Internet geht es um den berühmt-berüchtigten "Code", die digitalisierte Verkörperung von Verhaltensnormen in der Architektur des cyberspace. ${ }^{71}$ Dessen Entfesselung und Zügelung ist das Generalthema der Digitalverfassung parallel zur Entfesselung und Zügelung des Machtphänomens in der politischen Verfassung. Um Rechtsmaßstäbe zur Kontrolle des "Code" entwickeln zu können, bedarf es einer Analyse seiner rechtsspezifischen Risiken. Welche speziellen Gefährdungen individueller Autonomie ergeben sich aus der spezifischen Verhaltensregulierung durch den "Code"? Und wie wird die Autonomie gesellschaftlicher Institutionen vom "Code" tangiert? Sodann stellt sich die Frage, wie Verfassungsmaßstäbe, insbesondere die Grundrechte, internetadäquat zu rekonstruieren sind: Welche inhaltlichen und prozeduralen Kontrollen des "Code" sind nötig, um individuelle Grundrechte und institutionelle Bereichsautonomie gegenüber digitalen Verkörperungen von Rechtsnormen sichern zu können?

Dabei geht es gar nicht primär um Mißbrauch von digitalen Machtpositionen, sondern um die grundrechtsrelevanten Folgen der strukturellen Unterschiede von "Code" und Recht. Wenn eine Umorientierung der Verfassungsmaßstäbe angezielt ist, dann muß man sie an solchen Grundstrukturen des Internet ausrichten. Soweit der "Code" reicht, stellt er die normative Ordnung des symbolischen Raums Internet auf eine völlig neue Grundlage, weil das Verhalten der Netzteilnehmer nicht von sanktionsgestützten Verhaltensappellen der Rechtsnormen reguliert wird, sondern von den elektronischen Zwängen der Netzwerkprotokolle. Was bedeutet dann die Umstellung von traditionellen Rechtsnormen auf Netzwerkprotokolle für die Kommunikationsverfassung des Internet (Entscheidungen und Argumentationen) und für Autonomieräume individueller oder institutioneller Art (Grundrechte)? Was also sind die

\footnotetext{
${ }^{68}$ Zu einer institutionell verstandenen Grundrechtswirkung G e r s t e n b e r g , Oliver (1999) "Privatrecht, Verfassung und die Grenzen judizieller Sozialregulierung" In: Neumann, Ulfrid und Schulz, Lorenz (Hg.), Verantwortung in Recht und Moral. Stuttgart: Steiner,141-156; L a d e u r, Anm. 32; G r a b e r und T e u b n e r, Anm. 32.

${ }^{69} \mathrm{~W}$ alt e r, Anm. 11, 197.

${ }^{70}$ Dazu T e u bn er (2000b), Anm. 34.

${ }^{71}$ Lessig, Anm. 18; Reide nberg, Joel (1998) "Lex Informatica: The Formulation of Information Policy Rules Through Technology" Texas Law Review 76, 553-584.
} 
verfassungsrechtlichen Probleme des digital embodiment of law, der digitalen Verkörperung von Rechtsnormen?

Eine erste Antwort ergibt sich aus dem self-enforcing Charakter des Code. Was in der unter Internet-Juristen vorherrschenden instrumentalistischen Steuerungsperspektive als großer Vorteil des Code erscheint, ${ }^{72}$ wird zum Alptraum des Rechtsstaats. Traditionelles Recht beruht auf der institutionellen, verfahrensmäßigen und personellen Trennung von Rechtssetzung, Rechtsanwendung und zwangsweiser Durchsetzung. Das gilt weitgehend auch für Rechtssetzungsprozesse im privaten Sektor. Digitalisierung aber bewirkt nun eine Art Kernfusion von Rechtssetzung, Anwendung und Vollstreckung. Es verschwindet damit eine konstitutionelle Gewaltenteilung innerhalb des Rechtsprozesses und eine wichtige Garantie für individuelle und institutionelle Autonomiespielräume.

Einen zweiten Hinweis gibt die Trias von Verhaltenssteuerung, Erwartungsaufbau und Konfliktregulierung. ${ }^{73}$ Traditionelles Recht läßt sich nicht auf eine dieser Funktionen reduzieren, sondern verwirklicht alle drei, allerdings nur unter der Bedingung ihrer Dissoziierung, mit je eigenen Institutionen, eigener Rechtskultur und eigenen rechtsstaatlichen Sicherungen. Auch in dieser Trennung steckt eine (heimliche) Verfassungsgarantie gesellschaftlicher Autonomie. Denn hohe normative Anforderungen, die zur Klärung von Konflikten beitragen, müssen nicht notwendig als gesellschaftlich wirksame Erwartungen institutionalisiert werden, von ihrer Umsetzung in reales Verhalten ganz zu schweigen. In der digitalen Verkörperung von Rechtsnormen im Code aber wird diese Trias auf die einzige Funktion der elektronischen Verhaltenssteuerung reduziert. Damit gehen autonomiesichernde Pufferzonen zwischen Konfliktnormen, Erwartungsnormen und Verhaltensnormen verloren. Der Code des Internet bringt die zivilisatorischen Errungenschaften der Dissoziierung und die verfassungsmäßigrechtsstaatlichen Sicherungen der drei Dimensionen zum Verschwinden.

Kalkülisierung von Normativität ist ein weiterer verfassungsrelevanter Aspekt des Code. Traditionell war die vieldiskutierte Formalisierung des Rechts letztlich nur begrenzt möglich. Die Wirkungen, welche die Juristen am herkömmlichen Formalrecht schätzen oder fürchten, sind vergleichsweise harmlos gegenüber der im Code gelungenen Digitalisierung, die eine bisher ungekannte Formalisierung von Normen erzwingt. Denn die strikte Binarisierung $0-1$, die in der wirklichen Welt nur den Rechtscode im systemtheoretischen Sinne, also die Differenz Recht/Unrecht betraf, wird in der virtuellen Welt auf die Programme, also die gesamte Konditionalisierung der Entscheidung ausgedehnt. Das schließt, soweit der Internet-Code reicht, jegliche Interpretationsspielräume in den Programmen aus. Normative Erwartungen von Verhaltensweisen, die immer schon interpretiert, angepaßt, manipuliert, umgebogen werden können, wandeln sich zu rigiden kognitiven Erwartungen von faktischen Zuständen (Inklusion/Exklusion). Ein apokryphes Lernen, wie es in permanenten Mikrovariationen des Rechts durch neue Sachverhalte oder neue soziale Bewertungen stattfindet, ist im Code ausgeschlossen. Auch Rechtsargumente können im Anwendungsbereich des Code selbst keine Rolle mehr spielen. Argumente sind nur noch bei der Inkraftsetzung oder der offiziellen Änderung des Code zugelassen, nicht mehr aber in der juristischen Daueraufgabe der Auslegung, Anwendung, Durchsetzung von Normen. In der Sache bedeutet dies, daß jegliche Informalität im Anwendungsbereich des Code vollständig ausgeschaltet ist. Was im traditionellen Recht immer miterlaubt war, nämlich Ausnahmen vom Recht zu machen, Billigkeitserwägungen einzuschalten, das Recht schlicht zu umgehen, oder einfach auf nicht-rechtliche Kommunikation zu rekurrieren, funktioniert innerhalb des Internet-Code nicht. Die Digitalisierung erlaubt keinen informellen Dispens vom Code. Kein Wunder, daß in einer solchen Situation, die auf "brauchbare Illegalität" verzichten muß, die Figur des hacker, der den Code zu brechen versteht, geradezu zum RobinHood-Mythos wird.

Wenn dies die code-spezifischen Autonomiegefährdungen richtig kennzeichnet, dann wird deutlich, daß bestimmte rechtspolitische Forderungen an den Code durchaus verfassungspolitische Qualität besitzen. Die open-source-Bewegung, die bei jeder Vermarktung von Software strikt die Offenlegung des Quellcodes fordert, damit die Kontrollstruktur der Programme jederzeit nachprüfbar ist, erscheint dann nicht mehr bloß als eine Gruppe

\footnotetext{
72 In dieser instrumentalistischen Sicht unterscheiden sich die beiden Protagonisten der Internetverfassung nur wenig: L e s sig, Anm. 18, und J o hn s on und P o st, Anm. 18.

${ }^{73}$ Zu dieser Funktionstrias des Rechts, Anm. 4, $124 \mathrm{ff}$.
} 
sympathischer Idealisten. ${ }^{74}$ Oder: wenn Lessig fordert, daß die Digitalisierung von Verhaltenssteuerung über den Code immer vom Prinzip des narrow tailoring begleitet sein müßte, dann ist dies die Steigerung der Anforderungen des verfassungsrechtlichen Verhältnismäßigkeitsgrundsatzes gegenüber dem Code im Vergleich zur Rechtsnorm, die auch von Privatakteuren respektiert werden muß. Gerichtliche Überprüfungen, aber auch andere öffentliche Kontrollen von Meta-Normen des Code gewinnen dann eine Bedeutung, die über die vergleichbaren Kontrollen von Allgemeinen Geschäftsbedingungen oder Verbandssatzungen im wirklichen Raum weit hinausgehen. Das gleiche gilt für das Wettbewerbsrecht im Internet, da es nicht nur um die Öffnung von Märkten geht, sondern zugleich um das Offenhalten von alternativen Code-Regulierungen. ${ }^{75}$

\section{Viertes Merkmal: Dualverfassung von organisiertem und spontanem} Bereich

Wenn politisches Verfassungsrecht in der Sache zwei große Bereiche zu regeln hat Staatsorganisationsrecht und Grundrechte der Bürger - wie soll dies angemessen generalisiert und spezifiziert werden? Mein Vorschlag ist, daß es stets um die Normierung eines formal organisierten Bereichs und eines spontanen Bereichs innerhalb eines Teilsystems und besonders des prekären Verhältnisses zwischen ihnen geht. ${ }^{76}$ Eine demokratische Verfassung scheint davon abzuhängen, ob es gelingt, einen Dualismus von formal organisierter Rationalität und informaler Spontaneität als dynamisches Zusammenspiel ohne den Primat des einen oder des anderen zu institutionalisieren. In der Politik kommt es auf die wechselseitigen Kontrollen des formal organisierten Bereichs von politischen Parteien und staatlicher Verwaltung und des Spontanbereichs von Wählerschaft, Interessengruppen und öffentlicher Meinung an. Dies setzt sich in der Globalisierung im Verhältns zwischen Spontanbereich der internationalen Beziehungen und von internationalen Organisationen unter anderen Vorzeichen fort. In der Wirtschaft ist das Spannungsverhältnis von marktmäßig verfaßtem Spontanbereich und in Unternehmen verfaßtem Organisationsbereich - gerade nach dem neuesten Globalisierungsschub - fest etabliert. Auch in der weltweiten Forschung scheint es Tendenzen zur Entwicklung eines globalen Spontanbereichs gegenüber formalisierten Forschungsorganisationen zu geben. Im Erziehungssektor scheint der weltweite Wettbewerb von Universitäten die Rolle eines Spontanbereichs zu übernehmen. In all diesen Sektoren wäre es die verfassungsrechtliche Herausforderung, die Dualität gesellschaftlicher Autonomie in den Teilsystemen, also eine Kontrolldynamik von Spontanbereich und Organisationsbereich, auch normativ abzusichern.

Und im cyberspace zeigen sich ähnliche Entwicklungen. Lessig fürchtet eine Entwicklung des Internet zu unerträglicher Kontrolldichte durch eine Koalition ökonomischer und politischer Interessen. ${ }^{77}$ Während das Internet in seinen anarchischen Anfängen auf den Prinzipien der Inklusion aller, der Anonymität, Kontrollfreiheit und Heterarchie aufgebaut war, verstärken sich heute die politökonomisch motivierten Tendenzen zur Herausbildung von sogenannten Intranets, also geschlossenen Netzen, die auf Exklusion, Kontrolle, Hierarchie und strikter Zielorientierung beruhen. Die gleiche Entwicklung läßt sich aber auch anders, nämlich als Binnendifferenzierung des cyberspace in einen anarchischen Spontanbereich (Internet) und in verschiedene hochorganisierte Spezialbereiche (Intranets) interpretieren. Die Parallele zu anderen Sozialsystemen, in denen sich ein wechselseitiges Kontrollverhältnis von formal organisiertem Bereich und spontanem Bereich herausgebildet hat, ist deutlich. Und politisch würde es dann nicht darum gehen, mit Lessig u.a. die Entwicklung zum cybercorporatism zu bekämpfen, sondern darum, die Differenz spontan/organisiert als solche zu stabilisieren und institutionell abzusichern. Die Verfassung des Internet würde zwischen Spontanbereichen des Öffentlichen (ähnlich dem Grundrechtsteil des Grundgesetzes oder dem Marktverfassungsrecht) und hochformalisierten Organisationsbereichen (ähnlich dem Staatsorganisationsrecht oder dem

\footnotetext{
${ }^{74}$ L e s s i g, Lawrence (2001) The Future of Ideas. New York: Random House; B o y l e, James (2002) "Fencing Off Ideas" Daedalus, 13-25; Benkler, Yochai (2001) "Through the Looking Glass: Alice and the Constitutional Foundations of the Public Domain" Conference Paper, http://james-boyle.com

${ }^{75}$ L e s s ig, Anm. 18, 43 ff.

${ }^{76}$ Ausführlich T e u b ner (2000b), Anm. 34.

${ }^{77}$ Lessig, Anm. 18.
} 
Gesellschaftsrecht) unterscheiden, beide in ihrer Eigenlogik stabilisieren und würde ihre Hauptaufgabe darin sehen, ihre wechselseitigen Kontrollen ausbauen.

\section{Globale Zivilverfassungen in der Dichotomie öffentlich/privat}

Offensichtlich fügen sich globale Zivilverfassungen weder der Dichotomie öffentlich/privat noch der disziplinären Trennung von öffentlichem Recht und Privatrecht. Der Gegenstand zwingt das Völkerrecht und das transnationale Privatrecht bei der Verfassungsanalyse globaler, aber zugleich sektorialer Regimes zur Kooperation. Wie aber ist das Verhältnis von Arbeitsteiligkeit und Kooperation zu bestimmen? Eine disziplinäre Zuordnung je nach der "Staatsquote" des fokalen Regimes würde verfassungsrechtliche Zusammenhänge willkürlich zerreißen. Andererseits ist aber auch vom Konzept der international community, der Gemeinschaft der Völker, eine theoretische Führung nicht zu erwarten, da es eine globale Einheitlichkeit oder gar kollektive Handlungsfähigkeit suggeriert, die den heutigen Zustand einer fragmentierten Globalisierung nicht entsprechen. ${ }^{78}$ Vom Standpunkt der Systemtheorie bieten sich drei Gesichtspunkte an:

(1) Der Begriff der Weltgesellschaft ist im Unterschied zur international community von vornherein auf Dezentrierung angelegt: Die Weltgesellschaft ist eine Gesellschaft ohne Spitze und ohne Zentrum, die sich nur in ihren Fragmenten, eben den Verfassungen gesellschaftlicher Teilbereiche konstitutionalisieren lässt.

(2) Der Begriff des Weltrechts erlaubt es, von einem globalen Rechtssystem zu sprechen, das aber nicht als Einheit, sondern nur in fragmentierter Form besteht. Entscheidend ist, dass sich in den aktuellen Globalisierungsprozessen die traditionelle Binnendifferenzierung des Rechts verändert. Die Differenzierung in eine Vielheit nationaler Rechtssysteme wird überlagert von der Differenzierung in nicht territorial, sondern sektorial definierte Rechtsregimes, die zu den Trägern der Zivilverfassungen werden.

(3) Eine Integration gesellschaftlicher Teilverfassungen durch eine politische Gesamtverfassung ist nicht zu erwarten, wohl aber werden sich in der Kollision unterschiedlicher Teilverfassungen netzwerkartige Verknüpfungen der Verfassungen ergeben.

Von den jeweiligen begrifflichen Traditionen ausgehend wird sich bei aller Kooperation voraussichtlich eine schwerpunktmäßige Arbeitsteilung herausbilden, die dem Privatrecht die Aufgabe zuweist, die Eigenständigkeit und Autonomie gesellschaftlicher Teilverfassungen herauszuarbeiten, während sich das öffentliche Recht auf die Weiterentwicklung genuin politischer Verfassungen, auf die politischen Rahmenbedingungen von Zivilverfassungen und auf die netzwerkartige Verknüpfung der unterschiedlichen Teilverfassungen konzentrieren wird.

\footnotetext{
${ }^{78}$ Zum Begriff der internationalen Gemeinschaft T o m u s c h a t, Christian (1995) „Die internationale Gemeinschaft“ Archiv des Völkerrechts 33, $1 \mathrm{ff}$
} 